\title{
Provider-Patient Communication and Transition Readiness Among Adolescents with Type 1 Diabetes
}

Corrine N. Ahrabi-Nejad

West Virginia University, cna0006@mix.wvu.edu

Follow this and additional works at: https://researchrepository.wvu.edu/etd

Part of the Child Psychology Commons, Clinical Psychology Commons, and the Health Psychology Commons

\section{Recommended Citation}

Ahrabi-Nejad, Corrine N., "Provider-Patient Communication and Transition Readiness Among Adolescents with Type 1 Diabetes" (2019). Graduate Theses, Dissertations, and Problem Reports. 3830.

https://researchrepository.wvu.edu/etd/3830

This Thesis is protected by copyright and/or related rights. It has been brought to you by the The Research Repository @ WVU with permission from the rights-holder(s). You are free to use this Thesis in any way that is permitted by the copyright and related rights legislation that applies to your use. For other uses you must obtain permission from the rights-holder(s) directly, unless additional rights are indicated by a Creative Commons license in the record and/ or on the work itself. This Thesis has been accepted for inclusion in WVU Graduate Theses, Dissertations, and Problem Reports collection by an authorized administrator of The Research Repository @ WVU. For more information, please contact researchrepository@mail.wvu.edu. 
Provider-Patient Communication and Transition Readiness Among Adolescents with Type 1 Diabetes

Corrine N. Ahrabi-Nejad, B.A.

\author{
Thesis Defense submitted \\ to the Eberly College of Arts and Sciences \\ at West Virginia University \\ in partial fulfillment of the requirements for the degree of
}

Master of Science in

Psychology

Christina L. Duncan, Ph.D., Chair

Shari Steinman, Ph.D.

Claire St. Peter, Ph.D.

Department of Psychology

Morgantown, West Virginia

2019

Keywords: Type 1 Diabetes, Transition, Provider-Patient Communication

Copyright 2019 Corrine Ahrabi-Nejad 


\title{
Abstract \\ Provider-Patient Communication and Transition Readiness Among Adolescents with Type 1 Diabetes
}

\author{
Corrine N. Ahrabi-Nejad
}

The majority of adolescents with type 1 diabetes do not maintain a glycemic control within the recommended range. Poor diabetes control can yield both short term and long term acute health complications, making it critical for adolescents to achieve diabetes control. During this same time in development, adolescents are preparing for the transition from pediatric to adult diabetes care. Adolescents often transition to adult care based on their age rather than their transition readiness, which may result in a lack of support from their pediatric provider, potentially exacerbating their already poor glycemic control. Transitioning from pediatric care to adult care among adolescents with type 1 diabetes (T1D) often occurs spontaneously and with little guidance from pediatric providers. This abruptness in uncoordinated transition leads to decreased illness adherence. Research in the field of transition has identified that adolescents receive little information regarding the transition process including how to find an adult care provider, differences in adult care, and how to discuss their diabetes independently without a parent present. Adolescents who report poor transition readiness experienced gaps in care of 6 months or greater post-transition. Transition readiness can mitigate the negative effects that this transitional period often has. One potential method of increasing an adolescents' readiness to transition into adult care is through information disseminated by their pediatric provider. The current project assessed the relation between provider-patient communication and transition readiness in adolescents with type 1 diabetes. A total of 60 adolescents (ages 13 to 17) and their caregiver were recruited from West Virginia University Health Sciences Center. Participants were identified using the medical record database and eligible participants were approached and invited to participate at a regularly scheduled pediatric endocrinology clinic visit. Upon assent and consent, respectively, adolescents and their caregiver completed questionnaire measures using electronic tablets and all data were securely stored using REDCap software system. The aims of this study were to identify (1) whether adolescent better perceived provider-patient communication was associated significantly with greater transition readiness; and (2) if glycemic control acts as a moderator or mediator on this relation. Results from this study suggest that provider patient communication, as measured, is not a significant predictor of transition readiness, nor is glycemic control. Future studies should consider using alternative methods for measuring provider-patient communication, broadening sample characteristics for both providers and patients, and expanding investigation to include other factors that could potentially influence transition readiness. 


\section{Acknowledgements}

I would like to recognize and thank the many people who helped me throughout the entirety of this project. I would like to thank Dr. Christina Duncan, for her support in brainstorming research ideas, conducting data analysis, and creating this final report. I would also like to thank the members of my committee, Dr. Shari Steinman and Dr. Claire St. Peter, for both their time and thoughtful feedback on the development of this project. I would also like to thank the many undergraduate members of the Pediatric Lab for Adherence and Transition for their countless hours of time recruiting participants for this study. Finally, I would like to thank my family and friends for their unending support and encouragement. 


\section{Table of Contents}

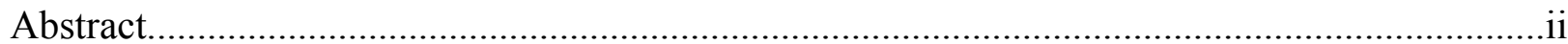

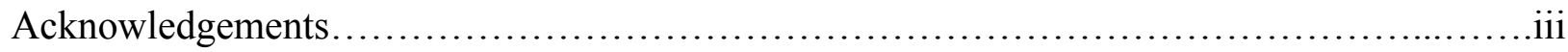

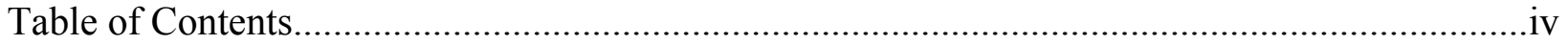

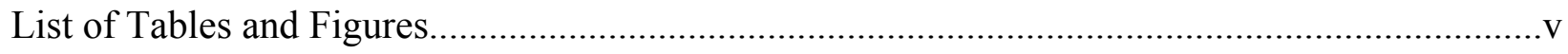

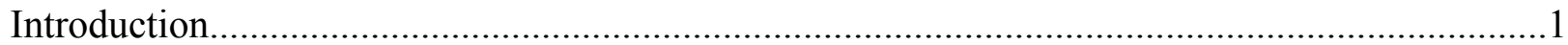

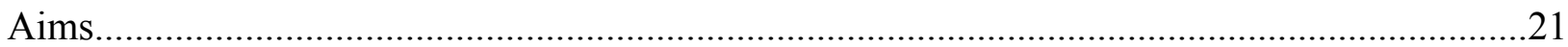

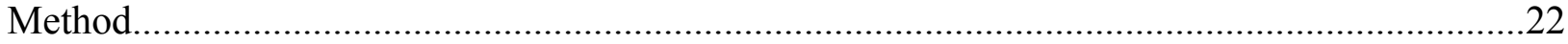

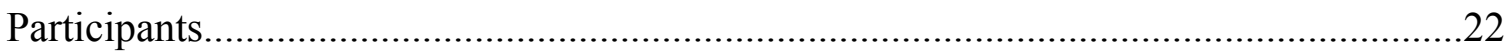

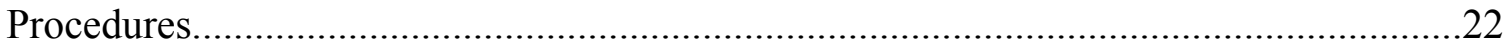

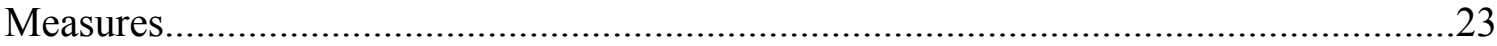

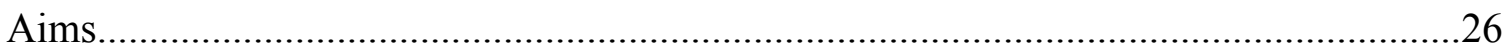

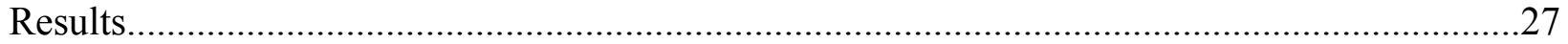

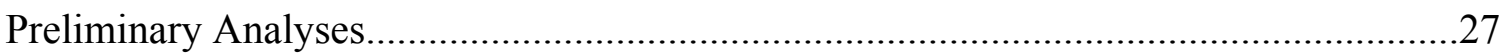

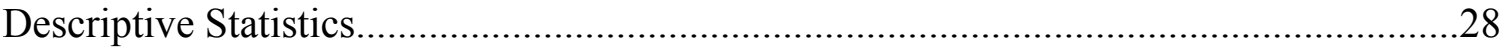

Effects of Provider-Patient Communication on Transition........................................29

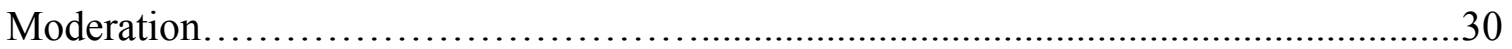

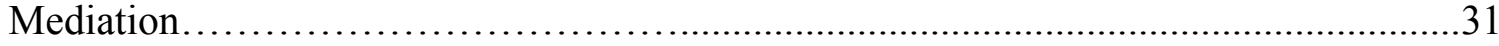

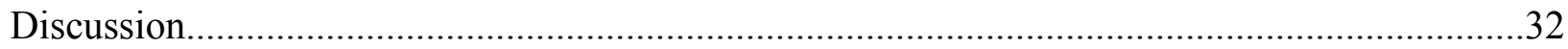

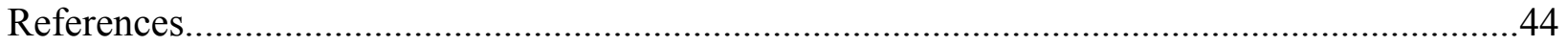

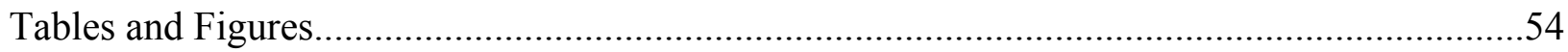




\section{List of Tables and Figures}

Table 1: Child and Parent Demographics Table................................................................54

Table 2: Descriptive Statistics and Correlations among Covariates and Variables.....................56

Table 3: Unstandardized Coefficients and Standard Errors for Regression Models..................57

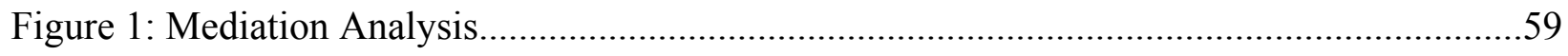

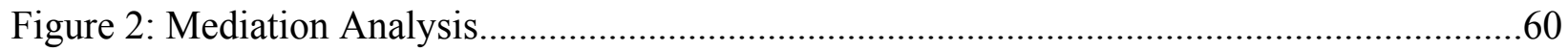

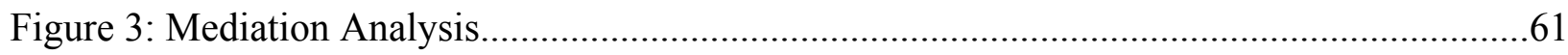

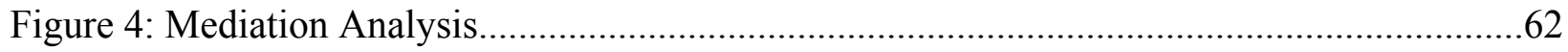




\section{Provider-Patient Communication and Transition Readiness Among}

\section{Adolescents with Type 1 Diabetes}

Type 1 diabetes (T1D) is a chronic illness that affects the pancreas' ability to naturally produce insulin (American Diabetes Association, 2017). Insulin is responsible for turning glucose into energy, and without insulin, high levels of glucose occur in the blood while the body starts to break down fat and muscle for energy (American Diabetes Association, 2017). Causes for T1D are still unknown; however, both genetic links and environmental factors are thought to contribute to T1D onset (Mayer-Davis et al., 2017). T1D typically is diagnosed in childhood and its incidence is rising. Indeed, recent studies conducted in partnership with the Center for Disease Control found a 1.8 percent increase each year of T1D (Dabelea et al., 2014; Mayer-Davis et al., 2017).

T1D requires intensive daily management to maintain healthy glycemic control (American Diabetes Association, 2017). Daily diabetes care involves insulin administration either via basal bolus insulin regimen (i.e., injection of insulin) or pump (automatically administers insulin through catheter), regular blood glucose monitoring, and dietary monitoring including calculations of carbohydrate intake while accounting for time and exercise (American Diabetes Association, 2015; Silverstein et al., 2005). Close monitoring of symptoms is required to identify acute or short-term diabetic complications such as hypoglycemia and DKA (diabetes ketoacidosis) (ADA, 2015b; ADA, 2015c; Silverstein et. al., 2005). Hypoglycemia comprises low blood glucose levels which can lead to seizures and unconsciousness, and DKA occurs when there is an inadaquate amount of glucose in the body for energy, resulting in burning fat for energy that leads to high levels of acidity in the body (ADA, 2015b; ADA, 2015c; Silverstein et. 
al., 2005). Failure to properly manage insulin levels and acute diabetic complications can lead to long-term health complications (e.g., blindness, amputation, death) (ADA, 2015).

Due to the complexity of these daily adherence tasks, during childhood, the majority of diabetes management is maintained or facilitated by parents or caregivers (Mellin, NeumarkSztainer, Patterson, \& Sockalosky, 2004; Pendley et al., 2002). As children mature, they begin to share responsibility with their caregiver to manage their T1D (Palmer et al., 2008). Typically, this process of transferring responsibility occurs around 8 to 10 years of age, leading to taking on the majority of responsibility in the teenage years (Lancaster, Gadaire, Holman, \& Leblanc, 2015; Palmer et al., 2008). Ideally during this transfer, caregivers slowly release control to their teen, while still providing the necessary support for T1D management (Lancaster et al., 2015). However, this period of transfer can have a negative impact on diabetes adherence and health behaviors, compromising glycemic control and increasing gaps in care between clinic attendance (Cadario, et. al., 2009; Logan et al., 2008). Therefore, adolescence is a developmental period needing further study to promote effective transfer of T1D responsibility.

\section{Transition of Care}

As adolescents assume more responsibility for their T1D care, the issue of transitioning medical care also becomes relevant. Transition of care within the medical contexts has been defined as "the purposeful, planned movement of adolescents and young adults with chronic physical and medical conditions from child-centered to adult-oriented health care systems" (Blum et. al., 1993, p. 570). National guidelines established by the American Diabetes Association (ADA) recommend that adolescents with T1D transition from pediatric to adult care sometime during the ages of 18-21 years (Lotstein et. al., 2013). Though all adolescents eventually transition to the adult care setting, a myriad of factors influence this transitional 
period for adolescents with T1D, with many individuals falling through gaps in medical care. The physical transition to a new medical provider, paired with the daily demands of T1D management as well as general psychosocial impacts of adolescence, poses many barriers to successful healthcare transition (Visentin, Koch, \& Kralik, 2006). Furthermore, physiological changes that occur during adolescence (e.g., hormonal changes) can negatively impact glycemic control, adding additional stressors on the adolescent (Bryden et al., 2001). As a result of the range of psychological, social, and physical factors associated with adolescence, glycemic control is poorest during the time period of 16 to 25 years of age, where the majority of adolescents are not within the recommended glycemic control parameters (Wills et al., 2003; Wood et al., 2013).

An additional challenge that adolescents face when transitioning is the vast difference in the function of a pediatric clinic compared to an adult clinic. While pediatric providers have a family-centric approach, adult providers have a patient-centric approach (Visentin et al., 2006). During childhood and adolescence, caregivers and children share T1D management. Thus, caregivers often attend clinic alongside their child and communicate regularly with the provider on the child's behalf (Visentin et al., 2006). However, in the adult care setting, patients typically attend clinic alone and have less direct support from providers and parents. Thus, an important aspect of the transition process is the gradual shift in diabetes management from parent shared management to self-management (Lyons, Libman, \& Sperling, 2013; Law, Kelly, Huey, \& Summerbell, 2002).

\section{Communication as a Protective Factor}

Though multiple factors impact transition, there exists limited research on what specific factors predict a successful transition into adult care among adolescents with T1D. Research 
among other health populations (e.g., HIV) found that optimizing provider-patient communication where providers educate adolescents on life skills related to disease management leads to increased self-management behaviors (e.g., making appointments, filling prescriptions), ultimately predicting improved transition to adult care (Cervia, 2013). These findings suggest that provider-patient communication may improve transition among other health populations, including T1D.

To address how communication impacts transition, other studies have also looked at adolescents' perspective of their provider's level of communication. An Australian-based qualitative study evaluated both provider and patient perspectives on the transition process, interviewing ten adolescents with T1D between the ages of 15 and 18 . Thematic coding revealed that indeed, adolescent report on provider characteristics indicates that providers often speak directly to parents, rather than adolescents, for the majority of the healthcare encounter when the parent attends appointments with their child (Lancaster et al., 2015; Visentin et al., 2006). A second qualitative study by Ritholz and colleagues (2014) conducted focus groups with 26 emerging adults with T1D. The focus group discussed provider characteristics which impacted the emerging adults' feelings towards transition. Thematic coding identified adolescents need for autonomy, as well as their desire for a more collaborative approach from their pediatric provider (Ritholz et al., 2014). With limited opportunities to communicate with their provider, adolescents may not develop the communication or educational skills necessary during their pediatric care to manage their diabetes independently in the future, particularly as they transition to adult healthcare (Visentin et al., 2006). Therefore, optimizing provider-patient communication, focusing on patient-centered communication can mitigate the negative impact of transition through self-management skill development. 


\section{Transition Readiness}

Transition readiness refers to the skills and knowledge necessary to successfully move into adult care (Lancaster et al., 2015). Though all adolescents partake in the transition process, readiness to transition varies between individuals and across time within individuals. Readiness to transition into adult care among adolescents with T1D involves sufficiently understanding their disease and its care, consistently engaging in daily disease self-management (e.g., insulin injections, blood glucose monitoring, carbohydrate calculations), and engaging in behaviors that promote long-term disease management (e.g., managing appointments, filling prescriptions, recognizing symptoms of hypoglycemia and hyperglycemia, and obtaining insurance) (Cervia, 2013). The range of transition readiness among adolescents makes it an important area to focus on as this readiness may predict their success in the transition process, yielding improved health behaviors when in adult care.

Based on the implications that transition readiness may have on health behaviors once in adult care, guidelines for pediatric providers have been established to support adolescents' transition. Guidelines include recommending an adult provider (along with the provider's contact information), coordinating an adult provider visit prior to full transition, providing written transition material, and assessing patient self-management skills (Garvey et. al., 2012; Simms, Baumann, \& Monaghan, 2017; Weissberg- Benchell, Wolpert, \& Anderson, 2007). Transition readiness among adolescents has been shown to positively correlate with transition satisfaction; however, an analysis of the literature reveals that the majority of pediatric providers are not facilitating a gradual release and are failing to prepare adolescents for the transition into adult care (Garvey et. al., 2012). Rather, transition often occurs because adolescents feel too old for pediatric care, because they move for college, or when they phase out of pediatric care based on 
age (i.e. after the age of 21). Adolescents are left responsible for finding a new provider, navigating the adult care system, and discussing their disease status with their new adult provider.

In summary, though all individuals eventually move into the adult medical setting, not all individuals exhibit the same degree of transition readiness when doing so. Though research is lacking regarding which specific factors improve transition readiness for adolescents with T1D, provider-patient communication is one area that is thought to improve transition readiness by helping adolescents develop communications skills needed in an adult care setting and aiding disease self-management skill development. The current proposed study aims to identify if provider-patient communication impacts this transition process among adolescents with T1D.

\section{Measures of Transition Readiness}

Since transition readiness has been identified as predicting transition success once in adult care, several clinical tools have been developed to measure individual transition readiness among chronic illness populations. For example, the UNC TrxANSITION assesses transition readiness for adolescents and young adult's ages 16 to 26 with a chronic health condition based on a 32-item questionnaire (e.g. "Do you usually have trouble remembering to take your medicines every day?”) (Ferris et al. 2012). Another commonly used tool for chronic illness populations is the Self-Management Skills Assessment, a 21-item assessment for children and adolescents 11 to 19 years old (Williams et al. 2010). The Self-Management Skills Assessment yields a strong Cronbach's alpha $(\mathrm{a}=0.89-0.93)$ and has a parent and child version, making it a common measure used in the field of research. However, despite the availability of transition measures, a systematic review found a general lack of reliability and validity among them (Stinson et al. 2013). In many of the existing measures of transition readiness, construct validity 
was not assessed. Additionally, factor analyses were not run, making it difficult to draw conclusions on the psychometrics of such measures and their predictive power (Zhang et al. 2014).

Though many measures exist to assess transition readiness, the Transition Readiness Assessment Questionnaire (TRAQ) is the most widely used based on its strong psychometric properties (Zhou, Roberts, Dhaliwal, \& Della, 2016). The $T R A Q$ measures transition readiness across a variety of chronic health conditions (e.g. diabetes, IBD, cystic fibrosis) (Jensen et al., 2017; Wood et al., 2014). Originally developed by Sawicki and colleagues (2011), the TRAQ is a self-report questionnaire consisting of 29 items completed by the adolescent to assess the extent of self-management behaviors (e.g., "Do you fill a prescription if you need to," "Do you manage your money \& budget household expenses? For example: use checking/debit card," "Do you keep home/room clean or cleanup after meals?'). Responses are recorded on a 5-point Likert scale based on the stages of change model: 1 "No I do not know how", to 5 "Yes, I always do this when I need to". A shorter 20-item version has since been developed and validated, along with a Cronbach's alpha of .94 documenting excellent internal consistency (Wood et al., 2014). The reliability and validity of the newer measure was assessed using a convenience sample of 447 adolescents from three medical clinics. Criterion validity was determined by testing for relationships between total $T R A Q$ scores for race, age, sex, and gender. This version is found to have acceptable criterion validity; however, further analysis is needed to identify its predictive validity (Wood et al., 2014).

\section{Factors Associated with Transition Readiness and Success}

The impact transition has on health behaviors makes transition readiness an important area to monitor, and is done so through the use of psychometrically validated tools. Across 
chronic illness populations, daily management in addition to long term health behaviors (e.g., filling prescriptions, following up with medical provider, accessing insurance) are needed to optimize health outcomes. Researchers in a Dutch study looked at the shortcomings of transition among 138 pre-transitioned adolescents, 181 of their parents, and 19 providers among 3 chronic illness populations (i.e., T1D, juvenile rheumatoid arthritis, and neuromuscular disorder with chronic ventilation) (Sonneveld, Strating, Van Staa, \& Nieboer, 2013). As part of a larger quality improvement study, adolescents who were receiving care through their pediatric provider, and their caregiver, each completed an online or paper questionnaire regarding provider characteristics and transitional care. Providers completed a similar measure, responding to Likert-type questions on the transition process, provider characteristics, and patient characteristics (Sonneveld et al., 2013). Findings indicated that adolescents were more satisfied with their transition experience compared to their parents; however, adolescents indicated lower rates of direct provider-patient communication (talking, listening, understanding, and honesty). Interestingly, providers did not identify provider specific characteristics (e.g., talking directly to the adolescent) as a barrier to transition but did identify lack of self-management by adolescents as well as parent over-involvement in care as a shortcoming (Sonneveld et al., 2013). This study shows the discrepancies in perceptions that exist between patients, their parents, and providers within the context of transition to adult care, and this incongruency may reduce transition readiness. Results suggest that in order to improve transition readiness, parents need to relinquish control, adolescents need to improve self-management, and providers need to enhance patientcentered communication (Sonneveld et al., 2013). It is important to note that glycemic control was not accounted for, which could be a potential moderator in their results. Moreover, generalizability of this study is limited by the European based sample, who receive healthcare 
services through a national healthcare system. Consequently, these findings may not generalize to a US-based healthcare system, in which access to affordable and dependable healthcare is moderated by health insurance status, for example.

A host of qualitative analyses have been conducted, highlighting the in depth complexities of transition to adult medical care across several health conditions. Patterson and Lanier (1999) conducted focus groups with 7 pre- and post-transitioned adolescents and young adults with a chronic illness or physical disability to identify the transition experience across health conditions. Thematic coding revealed similar barriers to successful transition across health conditions including not having a gradual transition experience, medical history not being transferred from pediatric care to adult care, limited independent self-management while still in pediatric care (i.e., parent over-involvement), and lack of knowledge on appropriate adult providers to which to transition. It is noteworthy that the study's sample was not described with respect to their specific health conditions. Although this study highlights specific concerns within the transition of care process across a range of health conditions, it is not specific to T1D, which may have unique circumstances. For example, the daily demands of managing T1D (i.e. blood glucose monitoring, insulin administration, carbohydrate monitoring) are different than those of other chronic illnesses, potentially influencing how adolescents experience the transition to adult care.

Indeed, several studies have explored the transition experience of adolescents with T1D and identified disease-specific barriers to successful transition as well as factors that are necessary for smooth transition. Garvey and colleagues (2012) conducted a study of posttransitioned young adults with T1D to identify characteristics that both aided and hindered transition into adult care. Questionnaires regarding the transition experience were mailed out to 
484 young adults, ages 22 to 30 years old, and of the 484 potential participants, 258 responded. Clinic records were used to collect corresponding HbA1c (glycated hemoglobin; a measure of the average blood sugar levels over the course of the previous several weeks). Results suggested that for the majority of young adults, transition was uncoordinated and often occurred for logistical reasons including moving for college, aging out of the pediatric healthcare system, and because the pediatric provider suggested transition (Garvey et al., 2012). Additionally, their findings indicated that over 50 percent of the young adults did not receive any transition education from their pediatric provider (Garvey et al., 2012). For example, missed opportunities for transition planning included attending a healthcare appointment without a parent, providerpatient discussion of screening tests that would be used in adult care settings, and provider recommendations for self-management. Furthermore, most providers did not give adolescents an adult provider recommendation or facilitate an integrated pediatric-adult provider appointment prior to transition (Garvey et al., 2012). This was the first cross-sectional, US-based study identifying transition characteristics for patients with T1D; its large sample size and national representation are its notable strengths. One important limitation was the high non-response rate from individuals on public insurance (i.e., Medicaid) as well as individuals with high A1C (>7.5), which ultimately resulted in a somewhat biased sample. Consequently, these study findings may not generalize to all individuals with T1D and in fact may imply an even larger gap in transition readiness among high risk adolescents with T1D.

In addition to quantitative studies, qualitative research also has delved into the complex factors associated with adolescents' transition into adult care. An Australian study by Visentin et al. (2006) examined patient (T1D) and provider perspectives on the diabetes transition process via focus groups and identified that few adolescents in the study knew differences in care 
between pediatric and adult settings. Additionally, interviews with adult providers revealed that based on provider perspectives, the majority of recently transitioned adolescents had little to no knowledge on how to manage diabetes sick days or ketoacidosis (Visentin et al., 2006). These findings suggest that limited communication between pediatric providers and adolescents regarding the transition process may be a significant factor in leaving adolescents unprepared for the demands of adult care.

Specific to T1D, poor transition readiness is not found to correlate with reduced glycemic control; yet, transition readiness has been correlated with diabetic adherence, as measured by frequency of blood glucose monitoring (Garvey et. al., 2012). Additionally, pre-transition HbA1c and diabetes adherence are the best predictors of future glycemic control (Garvey et. al., 2012; Jeffcoate, 2004). It is important to note that though puberty can negatively impact glycemic control for adolescents, engaging in positive care behaviors during adolescence is vital as these health behaviors predict future adult health behaviors (Garvey et. al., 2012). Adolescents who lack the necessary experience and skills for successful transition (e.g., attending clinic appointments individually, understanding screening test results, discussing factors for independent T1D management) are more likely to have a larger gap in care ( $>6$ months) following transition compared to their ready peers (Garvey et. al., 2012). Overall, transition should be approached as a process, gradually transitioning over time (e.g., at least one year prior to transition), involve integrated care between pediatric and adult providers prior to transition, and incorporate a gradual shift of responsibility from caregiver to adolescent to facilitate transition readiness (American Academy of Pediatrics, American Academy of Family Physicians, American College of Physicians, Transitions Clinical Report Authoring Group, 
2011; Garvey et al., 2016; Patterson, \& Lanier, 1999; Van Staa, Jedeloo, Meeteren, \& Latour, 2011).

The literature that exists has unanimously supported the need for structured transition protocols to yield a successful transition that maintains T1D adherence (Lyons et al., 2013). Published transition recommendations also establish clear guidelines for identifying transition readiness among adolescents with T1D (The Endocrine Society, 2013). Starting the transition process early is a key way to ensure adolescents develop the skills they need to be successful in adult care. During this time, providers can educate adolescents on how to independently navigate the adult-care medical setting, (e.g., identify insurance coverage, schedule appointments, and access their medication), assess adolescent self-management and diabetes knowledge, and clearly explain the differences in care in adult medical settings (e.g., adult medical providers do not automatically rebook when patients miss appointments; adult clinics have a heavy focus on complications screenings such as blood pressure and lipids monitoring ) (Lyons et al., 2013; Visentin et al., 2006).

\section{Transition Protocols}

Based on the importance of transition readiness among adolescents with T1D, research has aimed to identify the effects that structured transition protocols have on diabetes health behaviors and health outcomes. Logan et al. (2008) analyzed the effects of the Newcastle Diabetes Service in the United Kingdom (UK) on post-transition clinic attendance rates and diabetes health outcomes of 17- and 18-year-old adolescents over a 3-year period. The Newcastle Diabetes Service uses a step-based system in which adolescents gradually move from pediatric to adult care by first attending a joint pediatric-adult clinic and then attending a young adult clinic (Logan et al., 2008). Using the medical database system to assess clinic attendance and HbA1c, 
results show that the Newcastle Diabetes Service improved clinic attendance while not affecting HbA1c. Though glycemic control was still suboptimal in the majority of young adults, the authors posited that improved clinic attendance allows for increased services to be provided to reduce deterioration in the future (Logan et al., 2008). Additionally, improvement in diabetes self-management skills over the course of the three years may improve adherence, and in turn, glycemic control later in life. Absence of a control group, however, makes it difficult to draw conclusions on the effectiveness of the Newcastle Diabetes Service (Logan et al., 2008).

A similar study was conducted by Cadario et al. (2009), in which a retrospective analysis measured the effectiveness of a structured transition protocol over the course of 10 years on glycemic control, diabetes health behavior, and adolescent satisfaction with transition. Medical records of 62 adolescents (ages 18-20) with T1D who were transitioning into adult care were compared; nearly half the sample transferred to adult care without a structured protocol, while the other half received a structured transition (e.g., collaboration between pediatric and adult providers). Glycemic control and clinic attendance records were used to assess diabetes adherence and health behaviors pre- and post-transition. Additionally, questionnaires assessed adolescent satisfaction with transition. Results indicated that adolescents receiving the transition protocol had a shorter transition period compared to the control group (Cadario et al., 2009). Additionally, the same adolescents had lower HbAlc levels and higher clinic attendance compared to their unstructured transition counterparts (Cadario et al., 2009). Results contradict Logan et al. (2008), who did not find improved glycemic control with transition programming, but support other research (e.g., Lancaster et al., 2015; Logan et al., 2008; Visentin et al., 2006; Walleghem, MacDonald, \& Dean, 2008) indicating a decrease in the deterioration of key 
diabetes management behaviors. Consequently, findings across the extant literature are mixed with respect to the impact of structured transition programs on some key diabetes outcomes.

In contrast to a provider-based gradual transition protocol, “The Maestro Project” utilized a systems navigator model in which a transition specialist supported adolescents with T1D in their transition to adult care (Walleghem et al., 2008). A total of 84 adolescents were paired with a transition navigator at the start of their transition process. This experimental group was compared to a group of 64 post-transitioned young adults who had already transitioned without a transition navigator. Clinic attendance rates and HbA1c were compared across groups over the course of one year to identify the influence the transition navigator. Results found that adolescents who did not have a transition navigator at the start of transition experienced increased adult clinic dropout rates following transition. However, diabetes health outcomes did not differ across groups (Walleghem et al., 2008).

Several qualitative studies also have assessed the acceptability of transition protocols among adolescents with T1D and their providers. Step-based transition protocols have been implemented and evaluated in a few European countries. For example, the UK analyzed the effects of their "Transition Pathway" model, in which 11 adolescents with T1D in their final year of pediatric care received individualized transition education (Price et al., 2011). During this year, four one-on-one meetings were conducted, with the final meeting taking place in a “transition clinic" (for patients aged 16-25). During each session, qualitative interviews were used to evaluate the transition experience and readiness of the adolescent (Price et al., 2011). Thematic coding identified components important for transition, including the need for adolescent-focused healthcare and individualized treatment by providers (Price et al., 2011). The Parama Protocol was a similar British-based gradual transition protocol, in which 73 adolescents 
with T1D were gradually transitioned into adult care over the course of several months (Vanelli et al., 2004). During this time, adolescents received information on the transition process, were connected to an adult provider prior to transitioning, and attended a clinic visit jointly with their pediatric and adult provider (Vanelli et al., 2004). Individual interviews were conducted with each of the adolescents following the final transition visit to identify, retrospectively, the effects of the Parama Protocol. Qualitative analysis indicated that overall, adolescents found the Parama Protocol beneficial. Factors that led to positive transitions included appropriate transition age (20 years old), transitioning within the same medical system, adolescent and caregiver agreement on transition process (e.g., consensus on when to transition, who has T1D responsibility, etc.), contact with an adult provider prior to transitioning, cohesiveness between the pediatric and adult providers, and physician accessibility once the patient moves to adult care (Vanelli et al., 2004). Although this study's findings support the usefulness for transition protocols in enhancing the transition experience, this research did not evaluate the impact of the Parama protocol on diabetes adherence or health behaviors.

Current research suggests that structured transition protocols positively impact transition readiness among adolescents. However, it is important to note that many studies were conducted in Europe and Canada, which has a public healthcare system; therefore, the transition experience may not generalize to the American healthcare system, where insurance insecurity may have an added impact on transition. Additionally, feasibility and acceptability of a similar transition protocol applied in the U.S. may be hindered by other health care system differences (e.g., separate pediatric and adult provider medical settings, changes in insurance status). For example, the UK-based healthcare system already involves a "transition clinic" for youth aged 16 to 25 , which does not consistently exist in the United States. Overall, though these findings are quite 
promising, it will be important for transition programs to be developed for T1D and tested systematically with youth and young adults in the U.S.

Despite clear recommendations, analysis of international research has found that there is a relative lack in standardized transition protocol procedures for adolescents, resulting in uncoordinated and unplanned transitions (Fleming, Carter, \& Gillibrand, 2002; Rosen et al., 2003). Indeed, there are only a few randomized controlled studies that provide recommendations for evidence-based transition programs. Though existing research on transition programs in T1D highlight a generally positive impact on health behaviors (e.g., clinic attendance rates and gaps in care following transition), the paucity of systematic research makes it difficult to identify best practice models of transition as well as establish the true effectiveness of transition programs that exist (Lyons et al., 2013; Price et al., 2011; Rosen et al., 2003; Schidlow \& Fiel, 1990).

\section{Provider-Patient Communication}

Providing adolescents with the skills to independently manage their T1D is necessary for a successful transition, and provider-patient communication may be an effective channel to disseminate the knowledge needed to prepare adolescents for adult care. When pediatric providers prepare patients for transition, individuals report increased readiness and success in transition (Garvey et al., 2012). However, effective provider-patient communication is not achieved through just the provider, but also requires adolescents to have good skills to communicate with their physician and care team members.

Adolescents' ability to effectively communicate with their medical provider includes asking questions, accurately sharing information about diabetes management behaviors (e.g., frequency of insulin administration, blood glucose monitoring, and carbohydrate monitoring), and speaking openly and honestly about struggles with diabetes management and potential risk- 
taking behaviors (Simms et al., 2017). Higher rates of patient participation during clinic encounters positively correlates with degree of self-care and health outcomes (e.g., regimen adherence) for chronic illness management (Monaghan, Hilliard, Sweenie, \& Riekert, 2013; Simms et al., 2017; Street, Makoul, Arora, \& Epstein, 2009). Therefore, improving both provider and adolescent communication in the clinic setting could act as a protective factor against potential adverse effects during the transition process, by maintaining T1D adherence and healthy behaviors.

Unfortunately, several studies have revealed that adult providers find that many newly transitioned adolescents lack the self-management necessary to effectively communicate with them (Peters, \& Laffel, 2011). Though adolescents may desire the opportunity to self-manage, and self-managing will aid in their development of effective communication needed in the adult care system, other stakeholders such as parents and pediatric providers need to mutually relinquish control for this to occur. Provider communication targeting the adolescents while still in pediatric care can provide these youth with the communication skills necessary for adult care, making them readier for healthcare transition (Monaghan et al., 2013). Though provider-patient communication has been identified as important, no study to date has identified which provider characteristics are related to transition readiness in youth with diabetes. Consequently, the overarching goal of this study is to examine how pre-transitioned adolescents perceive communication from their endocrinology providers and ascertain which components of communication are most strongly predictive of transition readiness. Identifying a relevant measure of provider-patient communication will be key to achieving the study goal.

\section{Measures of Provider-Patient Communication}


Several tools have been developed to measure provider-patient communication in the clinic setting. A great deal of the literature uses qualitative measures to assess perceived provider-patient communication, applying them through interviews and focus groups (Rao et al., 2010; Simms et al., 2017). For example, the Four Habits Model is a qualitative measure used in research and clinical practice that measures provider communication (Krupat, Frankel, Stein, \& Irish, 2006; Rao et al., 2010). The four habits include: (1) Invest in the beginning; (2) Elicit the patient's perspective; (3) Demonstrate empathy; and (4) Invest in the end. Qualitative coding of the provider-patient interaction is used to assess the provider's degree of communication across these four areas. The Four Habits Model is found to have acceptable construct validity based on its correlation with the Roter Interaction Analysis System (RIAS) (Krupat, Frankel, Stein, \& Irish, 2006). However, the demand of the qualitative coding puts into question the feasibility of this measure to be used in routine clinic settings.

In contrast, some quantitative measures have been devised to assess provider-patient communication in the clinic setting. For instance, Campbell and colleagues (2007) developed a 19-item quantitative measure to assess both provider and patient perspectives on communication during clinic encounters (Campbell, Lockyer, Laidlaw, \& Macleod, 2007). Two parallel forms were developed, one for providers and another for patients to complete. Communication behaviors (e.g., "responded to your questions and concerns") are rated on a 5-point Likert scale ( $1=$ strongly agree, to $5=$ strongly disagree). Psychometric assessment with 1,845 doctor-patient dyads suggested good internal consistency ( $\alpha=.96)$ (Campbell et al., 2007). An exploratory factor analysis was run to assess validity of the measure, and two factors- process and content- were identified (Campbell et al., 2007). 
The Mind the Gap, another quantitative measure, was initially developed for juvenile idiopathic arthritis populations to assess parent and adolescent satisfaction with their medical providers (Shaw, Southwood, \& McDonagh, 2007). The Mind the Gap questionnaire assesses caregiver and adolescent perceived satisfaction with the medical provider using 3 themes: (1) provider related characteristics, (2) transition of care delivery, and (3) management of the medical environment. Adolescents and their caregiver(s) respond using a 7-point Likert scale. The Mind the Gap questionnaire has been psychometrically assessed on a juvenile idiopathic arthritis population, with a sample of 208 adolescents and 303 of their caregivers, and was found to be acceptable (Shaw, Southwood, \& McDonagh, 2007). Initial psychometric testing established content validity using existing qualitative data and literature for the specific chronic illness population. Construct and predictive validity were not examined (Shaw, Southwood, \& McDonagh, 2007). Internal consistency was found to be acceptable, with a Cronbach's alpha of $a=.91$ for adolescent report and $a=.94$ for parent report (Shaw, Southwood, \& McDonagh, 2007). The Mind the Gap has since been used among other chronic illness populations (e.g. diabetes) (Sonneveld et al., 2013). Because of its strong psychometric properties, its use among adolescents with a chronic illness, and its feasibility to be administered in a clinic setting, the Mind the Gap is a good tool to use when measuring provider communication.

\section{Summary and Rationale for Current Study}

Current research literature highlights the multidimensional complexities that surround T1D management and adherence for adolescents. Managing T1D is both physically and emotionally demanding, requiring daily blood glucose monitoring, insulin injections, carbohydrate monitoring, and maintaining adequate exercise (Silverstein et. al., 2005). Though diabetes management is typically shared between parents and children, caregivers often 
relinquish much of this responsibility to their adolescent (Lancaster et al., 2015; Palmer et al., 2008). The newfound autonomy of T1D daily management, paired with shifting social supports, hormonal changes, and preparing for the transition to adult care can be burdensome for many adolescents. In addition, glycemic control is worse among adolescents, where less than 75 percent of adolescents are able to maintain a glycemic control within the recommended range (Wills et al., 2003; Wood et al., 2013). At the same time, health behaviors in adolescence are the best predictors for future health behavior (Garvey et. al., 2012). A multitude of factors can be barriers to T1D adherence, which can lead to both short-term (e.g., hypoglycemia) and long-term complications (e.g., blindness, amputation, death) (ADA, 2015). Therefore, to optimize healthcare transition success and subsequent positive health outcomes, it is critical to understand factors that affect transition readiness in adolescents with T1D.

A review of existing transition protocols has revealed varying findings regarding their effectiveness in promoting successful transition, adherence, and health outcomes in T1D. Some studies have found transition protocols to have little effect on T1D health outcomes (e.g., Lancaster et al., 2015; Logan et al., 2008; Walleghem et al., 2008). Other studies have found transition protocols to improve health behaviors following transition, including regular clinic attendance (e.g., Cadario et al., 2009; Lancaster et al., 2015; Logan et al., 2008; Visentin et al., 2006; Walleghem et al., 2008). Divergent results may stem from factors such as different content and timing of transition programs, non-uniform outcome measures, and varied patient samples. Consequently, no unanimous conclusion has been made regarding how transition protocols enhance transition readiness; thus, more research is needed to identify barriers and facilitators to transition readiness for adolescents with T1D. 
Patient perception of provider communication is one potential barrier or facilitator to consider. Provider-patient communication provides both the information necessary for transition (e.g., self-management skills, adult provider recommendations) and the requisite support (Simms et al., 2017). Furthermore, enhanced provider-patient communication correlates with better health outcomes among adolescents with T1D (Simms et al., 2017; Street et al., 2009). However, discrepancies exist between provider and adolescent reports on the quality of communication that occurs, with adolescents reporting poorer quality of communication (Sonneveld et al., 2013). Specifically, based on previous research, it is thought that provider communication may improve transition readiness among adolescents with T1D; however, it has not yet been studied. As a result, the overall goal of this project was to examine the relation between adolescent perceptions of provider-patient communication and their ratings of transition readiness. Specific aims are as detailed below.

Aim 1. The first aim of this study was to examine the association between perceived provider-patient communication and transition readiness. Based on transition literature, which found provider communication to be associated with patient empowerment (Sonneveld et al., 2013), it was hypothesized that more positive reports of provider patient communication by adolescents and parents would be associated with increased transition readiness.

Aim 2. The second aim of this study was to explore whether glycemic control acts as a moderator or a mediator on provider-patient communication predicting transition readiness. Adolescents with poor health behaviors may not regularly attend clinic or may not actively engage in their clinic encounters (e.g., attend to their phone, listen to music through headphones), potentially decreasing their satisfaction with provider communication and their readiness for transition. Understanding the specific relations among these variables has the 
potential to further inform efforts to devise effective transition programming. Because there is no literature that has examined these variables together, no hypothesis was proposed.

\section{Method}

\section{Participants}

A total of 60 adolescents with T1D and their parent/primary caregiver were recruited from pediatric endocrinology clinics at West Virginia University Health Sciences Center in Morgantown, West Virginia. This study was part of a dissertation project involving investigation of health literacy, transition readiness, and adherence in adolescents and young adults with T1D. Inclusion criteria included that adolescents were: (a) between the ages of 13 and 17 years; (b) having been diagnosed with T1D for at least one year; and (c) having a caregiver who was willing to participate. Participants with cognitive deficits or language barriers (identified by the medical team) were not included in the study, as these barriers may lead to difficulty comprehending and completing study measures. Additionally, participants with comorbid medical conditions (i.e., cystic fibrosis) were not included in the study, as additional complex medical conditions may influence their interactions with medical providers.

\section{Procedure}

The overarching dissertation project was two phased. Using quantitative questionnaires, Phase 1 assessed the relation between health literacy, transition readiness, and diabetes health outcomes. Additionally, analysis of how family factors act as a moderator on this relation was measured. Phase 2 employed qualitative focus groups to understand how beliefs and perceptions of health literacy and family factors influenced the transition process. IRB approval was obtained prior to the start of data collection. Recruitment of participants began in January 2018, with a minor amendment adding new study measures in December 2017. 
The current study recruited participants during Phase 1 of the overarching dissertation project. Participants who met the age and diagnosis inclusion criteria were recruited when they attended a routine appointment in the pediatric endocrinology clinic. Interested patients and families were given a description of the study, their responsibilities if taking part, and potential risks. Research staff then answered the family's questions. When a patient and their caregiver were interested in participating in the study, assent and consent were obtained, respectively.

Study questionnaires were administered as a battery, with the same order of presentation across all participants (order appears in the description of measures below). Parents completed demographic questionnaires and parent forms. Following this, adolescents completed the remainder of the questionnaires. Questionnaires were completed using a tablet and responses were logged using the REDCap software system. This system securely stores all participant data. Participants were provided a paper version of all questionnaires, if requested, as an alternative to the tablet system; however, all participants enrolled in the study elected to complete the questionnaires using the tablet. Upon completion of study measures, adolescents were compensated for their time in the form of a $\$ 20$ gift card.

\section{Measures}

Family Information Form. The Family Information Form collected demographic and medical information about the patient and family including age, race, education, SES, and medical background. The form was completed by the caregiver.

Transition Readiness Assessment Questionnaire (TRAQ; Wood et al., 2014). The $T R A Q$ is a measure of transition readiness, which measures an individual's ability to self-manage specific health related tasks. The 20 -item scale is completed by adolescents and consists of 5 subscales: (1) Appointment Keeping, (2) Managing Medication, (3) Talking with Providers, (4) 
Tracking Health Issues, and (5) Managing Daily Activities. Respondents answer the items using a 5-point Likert scale: 1 = "I do not know how to do this;" 2 = No but I want to learn;" 3 = "No but I am learning to do this;" 4 = Yes I have started doing this;" and 5 = "I always do this when I need to". A total transition readiness score is calculated by averaging all 20 items. Subscale scores for each of the 5 subscales are also obtained by averaging all items in each subscale. The $T R A Q$ has strong psychometrics and yielded strong internal consistency (Cronbach's $\alpha=.94)$ when used with adolescents with health-related needs (Sawicki, et al., 2011; Wood et al., 2014). Strong reliability was obtained using Cronbach's alpha, for 4 of the subscales ranging from .77 to .90 (Wood et al., 2014).

Total $T R A Q$ scores were used in analyses to identify general transition readiness across all 5 domains. A total score minus the "Talking with Providers" subscale (i.e., TRAQ without Communication subscale) also was calculated for separate data analysis. Consequently, this score removed the overlap in similar items between the "Talking with Providers" subscale and the TRAQ. Larger scores are indicative of higher transition readiness. Responses on the TRAQ indicated good internal consistency with a total score Cronbach's Alpha of .92. For the TRAQ without the Communication subscale score, internal consistency also was excellent (.93).

\section{Mind the Gap (Shaw, Southwood, \& McDonagh, 2007). The Mind the Gap}

questionnaire assesses caregiver and adolescent perceived satisfaction with the T1D medical provider, by considering the medical environment, provider characteristics, and process issues [e.g., "provides information to other professionals involved in my health care (e.g., family doctor)"]. Participants rate their current level of care, responding to items using a 7-point Likert scale ranging from 1 (strongly disagree) to 7 (strongly agree). The adolescent version contains 22 items, while the parent version contains 27 items. A total score for both the adolescent and parent 
version, which is calculated by averaging all item responses, was used in this study's analyses. Additionally, a total subscale score of the "Provider Characteristics" scale (11 items for the adolescent version;14 items for the parent version) was used for separate data analysis. Higher scores indicate higher reports of satisfaction with the provider (total score) and provider-patient communication (Provider Characteristics scale).

The Mind the Gap questionnaire has demonstrated reliability and validity in measuring perceived satisfaction of medical care among children and parents within a medical setting (i.e., juvenile idiopathic arthritis) (Shaw et al., 2007). Psychometric testing was run using 301 adolescents with juvenile idiopathic arthritis and 286 caregivers. Internal consistency was determined to be satisfactory, indicated by a Cronbach's alpha of .91 for adolescents and .94 for caregivers (Shaw et al., 2007). Because of the acceptable psychometric properties, as well as the benefits of having both parent and child report versions, the proposed study used the Mind the Gap to measure provider-patient communication. In the current study, responses on both adolescent and parent version of Mind the Gap indicated good internal consistency with a total Cronbach's Alpha of .94 and .97, respectively, for the total score. For the Provider Characteristics subscale score, internal consistency also was excellent (.90 for adolescent; .97 for parent).

\section{Medical chart review.}

HbA1c. The date and value of the most recent HbA1c was collected from the patient's medical record. HbA1c is typically measured through routine blood work every 3 months for adolescents with T1D (Jeffcoate, 2004). The value measures the patient's current blood glucose level and is a function of blood glucose levels over the previous 2 to 3 months. An ideal HbAlc level for adolescents with T1D is 7.5\% and below (ADA, 2017). 
Insurance status. Current insurance status was collected from the patient's medical record and coded as a categorical variable (i.e., Medicaid vs. private insurance).

Amount of time with provider. As a proxy for time with provider, the participant's medical record was reviewed to summarize the number of contacts in person (i.e., clinic appointment; hospitalization) with the provider over the previous 12 months.

Aim 1. The first aim was to examine the association between perceived provider-patient communication and transition readiness among adolescents with T1D. To measure this relation, a Pearson correlation coefficient was used to assess how provider-patient communication (Mind the Gap) relates to transition readiness (TRAQ). Separate correlations were calculated, using both total scores from the TRAQ and Mind the Gap, as well as their respective subscale scores. The first correlation was run using the $T R A Q$ total score and Mind the Gap total score. To account for the potential high correlation between Mind the Gap and TRAQ due to the Communication subscale, a second correlation was calculated using $T R A Q$ without Communication subscale and Mind the Gap Provider Characteristics subscale. When covariates (e.g., gender, age, race, insurance status, length of time with provider) were identified, a partial correlation was calculated, controlling for the identified covariates.

Aim 2. The second aim was to explore how glycemic control moderates or mediates the relation between provider-patient communication (Mind the Gap) and transition readiness (TRAQ). Two separate moderation analyses and two separate mediation analyses were performed using total scores from $T R A Q$ and Mind the Gap as well as TRAQ score (without Communication subscale) and Mind the Gap Provider Characteristics subscale score. Moderation and mediation analyses were performed using PROCESS. Bootstrapping was used based on 10,000 bootstrap sample, as is recommended with smaller sample sizes (Preacher \& Hayes, 2008). When 
interpreting the results, statistical significance was set at the .05 level, as is indicated in related social science research.

\section{Results}

\section{Preliminary Analyses}

Statistical analyses were run using Statistical Package for the Social Sciences Version 23 (SPSS 23).

Power Analysis. An a priori power analysis was conducted using G.Power 3.1.9.2. (Erdfelder, Faul \& Bechner, 1996). For Aim 1, the statistical test was entered as a correlation: bivariate normal model. Input parameters were a one-tailed test, with correlation of 3 (medium effect size), alpha value of 0.05 , power of .80 , and correlation of null $\mathrm{H} 0$ as 0 . To detect a medium effect size, a sample of 67 youth is required. To detect a large effect size (.5) with the same alpha and power levels, a sample of 23 is required. It is noteworthy that effect sizes were not reported in the relevant literature.

Aim 2 involved a mediation and moderation analysis. It has been argued that a power analysis for these types of analyses are not necessarily appropriate (Thoemmes, MacKinnon, \& Reiser, 2010). However, for the sake of this study, a power analysis was conducted for a linear multiple regression to estimate the sample size. Using a medium effect size (.20) with an alpha of .05 and power of $.80,60$ participants was found to be adequate. Consequently, the current study, with a sample of 60 participants, appears to be adequately powered to detect a medium effect size.

Missing Data. Preliminary analysis was conducted to identify any missing data. Four participants were missing more that $10 \%$ of items on a given measure, and were therefore excluded from analyses. Following the removal of the four participants, the remaining 56 
participant's data was assessed. Little's MCAR test identified that all missing data in this sample was missing completely at random, $X^{2}(735, N=56)=618.00, p=1.00$. Imputation based procedure using expectation-maximization (EM) was applied to account for the missing data.

Assumption Checks. Assumptions of skew, kurtosis, and normal distribution, were assessed prior to conducting statistical analyses. Parent Mind the Gap scores and Adolescent Mind the Gap scores were not found to be skewed (skew $=1.9, \mathrm{SD}=.62$; skew $=3.1, \mathrm{SD}=.84$ ), and no transformation was needed. Univariate outliers were assessed by converting questionnaire scores into z-scores. Upon assessment of univariate outliers among the variables, one participant had a z-score of 3.58, which indicates that this participant may bias results. This potential issue was resolved by running analyses with and without the participant; when doing so, no differences were noted, indicating the outlier did not significantly impact the data. Additionally, correlations of all dependent variables were found acceptable $(<.60)$, suggesting no bivariate outliers.

\section{Descriptive Statistics}

Descriptive statistics for all variables are provided in Table 1. A total of 60 adolescents and their primary caregiver participated in the study. Due to missingness, 56 participants were included in the current analysis. Approximately half of the participants were male $(56 \%)$ with the majority of caregivers were mothers (80\%). Adolescents were between the ages of 13 to 17 years old, with a mean age of 15.5 years $(\mathrm{SD}=1.5)$. Adolescents in the study had an average HbA1c of 8.65 with a standard deviation of 1.77 , which is above the recommended range $(<7.5)$ for individuals with T1D. During the enrollment period, 60 participants consented to participate in the current study and 47 refused. Reasons for refusal included lack of time, no interest in participating in a study, and needing additional lab work immediately following the clinic 
appointment. Both adolescents $(M=6.15, S D=.84)$ and their caregivers $(M=6.27, S D=.62)$ reported a positive level of provider-patient communication, though their reports were not significantly different $[t(55)=.93, p=.36] . T R A Q$ scores were also analyzed to determine the degree of transition readiness. The majority of adolescents reported a moderate level of transition readiness $(M=3.38, \mathrm{SD}=0.83)$, corresponding to the Likert rating anchor of "No, but I am learning to do this."

\section{Covariates}

Pearson's correlation coefficients were calculated to assess potential covariates in the model, with a focus on participant age, gender, time with provider, duration of T1D diagnosis, and insurance status. Participant age positively correlated with transition readiness $(r=-.42, p=$ .002), indicating that older participants were more likely to report better transition readiness. Additionally, glycemic control was positively associated with length of diabetes duration $(r=$ $.35, p=.02)$, indicating that participants who have had T1D longer are more likely to have elevated HbA1c levels. No other covariates were identified in the dataset. Analyses in the current study were run controlling for participant age and length of diabetes duration (Hayes, 2017).

\section{Aim 1 Analyses: Effects of Provider-Patient Communication on Transition Readiness \\ Pearson's Correlation. Partial correlations were assessed between Mind the Gap and $T R A Q$ scores, controlling for age and duration of diagnosis. Pearson correlation coefficients between Mind the Gap and TRAQ scores was not significant for adolescents $(r=-.12, p=.38)$ or caregivers $(r=.09, p=.50)$. To account for a potential correlation due to the similarities of the two scales, a second correlation was calculated using $T R A Q$ without Communication subscale and Mind the Gap Provider Characteristics subscale. Correlations between Mind the Gap}


Provider Characteristics subscale and $T R A Q$ without Communication subscale scores was not significant for adolescents $(r=-.15, p=.29)$ or caregivers $(r=.09, p=.50)$.

\section{Aim 2 Analyses: Effects of Glycemic Control on Provider-Patient Communication and}

\section{Transition Readiness}

Moderation. Mediation and moderation analyses were run controlling for participant age and length of diabetes duration using PROCESS (Hayes, 2017). Glycemic control was examined as a moderator between self-reported satisfaction with provider-patient communication (Adolescent Mind the Gap) and transition readiness (TRAQ). The interaction term between glycemic control and self-reported provider-patient communication did not explain a significant amount of variance in transition readiness, $\Delta R^{2}=0.07, F(5,41)=0.63, p=0.67$. Therefore, glycemic control was not a significant moderator of the relation between self-reported satisfaction with provider-patient communication and transition readiness.

Glycemic control was examined as a moderator between parent-report of satisfaction with provider-patient communication (Parent Mind the Gap) and transition readiness (TRAQ). The interaction term between glycemic control and parent-reported provider-patient communication did not explain a significant increase in variance in transition readiness: $\Delta R^{2}=$ $0.02, F(5,41)=0.21, p=0.96$. Consequently, glycemic control was not a significant moderator of the relationship between parent-reported provider-patient communication and transition readiness.

To account for potential correlations between provider-patient communication and transition readiness due to similar items in the scales, a separate moderation analysis was run using the Adolescent $M T G$ Provider Characteristics subscale and the $T R A Q$ without Communication subscale. The interaction term between glycemic control and self-reported 
provider-patient communication did not explain a significant increase in variance in transition readiness, $\Delta R^{2}=0.07, F(5,41)=0.57, p=0.72$. Additionally, the interaction term between glycemic control and parent-reported provider-patient communication did not explain a significant increase in variance in transition readiness, $\Delta R^{2}=0.02, F(5,41)=0.14, p=0.98$. As a result, glycemic control was not a significant moderator of either relationship. The interactions can be seen in Table 3 .

Mediation. Glycemic control was then examined as a possible mediator between selfreported provider-patient communication (Adolescent $M T G)$ and transition readiness (TRAQ). When entered into the regression analysis, $\Delta R^{2}=0.01, F(4,42)=.14, p=0.97$, CI $[-0.09,0.06]$ indicating that the relationship between self-reported provider-patient communication (Adolescent $M T G$ ) and transition readiness $(T R A Q)$ is not mediated by glycemic control. This interaction is displayed in Figure 1.

Glycemic control was then examined as a mediator between parent-reported providerpatient communication (Parent $M T G$ ) and transition readiness (TRAQ). When entered into the regression analysis, $\Delta R^{2}=0.02, F(4,42)=.21, p=0.93$, CI $[-0.14,0.14]$ indicating that the relationship between parent-reported provider-patient communication (Parent $M T G$ ) and transition readiness $(T R A Q)$ is not mediated by glycemic control. This interaction can be seen in Figure 2.

In order to address potential correlation due to similarities of the two scales, a separate moderation was run using the Adolescent $M T G$ Provider Characteristics subscale and the TRAQ without Communication subscale. Results indicated that glycemic control did not significantly mediate the relationship between self-reported provider-patient communication and transition readiness $\left(\Delta R^{2}=0.02, F(4,42)=0.21, p=0.93, \mathrm{CI}[-0.11,0.07]\right)$. This interaction is shown in 
Figure 3. In contrast, results revealed a significant effect between glycemic control and parentreported provider-patient communication $\left(\Delta R^{2}=0.24, F(3,43)=4.6, p=0.007\right)$. Specifically, more positive provider-patient communication appears to be associated significantly with better HbA1c levels. Nevertheless, glycemic control did not mediate the relationship between providerpatient communication and transition readiness $\left(\Delta R^{2}=0.006, F(4,42)=.08, p=0.99, \mathrm{CI}[-0.28\right.$, 0.32]). Figure 4 shows this interaction.

\section{Discussion}

The transition from pediatric to adult care for adolescents with T1D can be a challenging period to navigate, and health outcomes (e.g., glycemic control) can be influenced by an adolescent's readiness for that transition. Given the impact transition readiness has on health behaviors for adolescents with a chronic illness, it is important to identify factors that may improve transition readiness. This study aimed to examine (1) the extent to which providerpatient communication predicts transition readiness among adolescents with $\mathrm{T} 1 \mathrm{D}$, and (2) whether glycemic control moderates or mediates the relationship between provider-patient communication and transition readiness. Research to date has not yet characterized the relation between provider-patient interaction and transition readiness. Consequently, our study contributes to the current literature by investigating clinically meaningful areas that might be optimized through intervention in future research, which in turn could improve transition readiness for adolescents with $\mathrm{T} 1 \mathrm{D}$.

In general, our results did not suggest that adolescent provider-patient communication significantly predicts transition readiness among adolescents with T1D. Interestingly, the standardized regression coefficient between parent-reported provider-patient communication subscales and glycemic control was statistically significant $(b=-1.44, p=.01)$. This finding 
suggests that better parent-reported provider-patient communication is significantly associated with better adolescent glycemic control. Nevertheless, these significant findings are not consistent with other current results and seem to be a chance occurrence. Specifically, providerpatient communication did not significantly predict glycemic control in the other three mediation analyses. Further, no relationship was found between either parent-reported or adolescentreported provider-patient communication and transition readiness. Our nonsignificant findings suggest that transition readiness may be better predicted by other factors outside of provider communication. Indeed, the current study did not assess factors such as adolescent attitudes towards transition, adolescent autonomy or responsibility for diabetes care, or level of patient self-efficacy, all of which could be relevant to transition-readiness. Additionally, given the current sample's ceiling effect in the provider-patient communication measure, sample characteristics as well as measurement shortcomings could account for our nonsignificant results.

Transition readiness could be associated with other factors that were not measured in the current study, such as adolescent attitude towards transition and adolescent degree of selfefficacy (Van Staa, Van der Stegege, Jedeloo, Moll, \& Hilberink, 2010). Previous research found that adolescents who report more negative attitudes (e.g., fear) towards the transition process reported poorer transition readiness (Van Staa et al., 2010). The current study did not assess adolescent's attitudes towards transition, which could be a potential mediating factor in the relationship between perceived patient-provider communication and adolescent transition readiness. Qualitative interviews (e.g., "What are some positive aspects of transition?" "How can providers best support you in making the transition from pediatric to adult care?"), for example, can be used to identify individuals who have more negative attitudes towards transitioning (Van Staa et al., 2010). Further, adolescent-self efficacy, or the degree to which the adolescent feels 
able to manage his or her own care successfully, is thought to be predictive of transition readiness (Van Staa et al., 2010). The Diabetes Management Self-Efficacy Scale

for Adolescents with Type 1 Diabetes, which has been found to be an acceptable measure of selfefficacy for adolescents with T1D, could be used in future research to assess self-management behaviors for a variety of diabetes specific tasks (Moens, Grypdonck, \& Van, 2001). Overall, non-significant results in the current study suggest that future research could benefit from exploring other pertinent constructs alongside provider-patient communication to better understand transition readiness in youth with T1D.

A second factor potentially impacting the results of the current study is its sample characteristics. Our sample was rather homogenous for both patient and providers, with $96 \%$ of patients and $100 \%$ of providers identifying as Caucasian. The provider-patient interaction and self-reported satisfaction with provider-patient communication may be impacted by the same race provider-patient dyad (Aronson, Burgess, Phelan, \& Juarez, 2013). Past research has found that minority patients report briefer, and less warm interactions when interacting with providers from majority groups (Aronson et al., 2013). Ethnic and racial minority patients also report less trust in their medical providers, when compared to their Caucasian counterparts, likely limiting provider-patient communication (Armstrong, Ravenell, McMurphy, \& Putt, 2007). Additionally, studies identifying ecological factors impacting transition readiness identified race as one factor, with non-white patients having lower transition readiness (Javalkar, et al., 2016). It can be speculated that poorer provider-patient interaction may prevent minority patients from developing skills needed for self- management, ultimately negatively influencing their transition readiness. Therefore, results from the current study may not be indicative of provider-patient 
communication ratings for non-white participants, and therefore cannot generalize to the broader population.

Moreover, not only does the racial and ethnic make-up of the provider-patient dyad have a significant relation to provider-patient communication, but the provider's cultural competence has also been shown to be associated with patient's attitudes towards their care (Monaghan, Hilliard, Sweenie, \& Riekert, 2013). Research has identified cultural differences in how individuals interact with their health care environment, with African American patients preferring to play an active role in medical decision making (Peek et al., 2008). Cultural differences also impact non-verbal communication including tone, eye contact, and how one interacts with a person of authority (i.e., physician) (Teal \& Street, 2009). In turn, these cultural differences in non-verbal communication style may negatively impact the patient's satisfaction with the provider-patient communication and lead to worse transition readiness. No study has yet to look at how cultural differences impact provider-patient communication among adolescents with T1D, or how cultural differences in communication influence transition readiness.

Therefore, it is necessary to study the satisfaction with provider-patient communication among culturally and ethnically diverse patient and provider populations to better understand processes of communication that may be racially and culturally impacted.

Previous research has identified other provider factors, beyond cultural competence, that potentially impact patient satisfaction; these factors include provider medical knowledge and provider flexibility towards medical decision making (Lugasi, Achille, \& Stevenson, 2011). Such provider characteristics, in turn, affect patient satisfaction, and could also influence degree of effective communication. Research has found provider characteristics, such as tone, affect, information sharing, and promotion of patient autonomy are positively associated with health 
care satisfaction and reduce health care complications (Monoghan, Hilliard, Sweenie, \& Riekert, 2013) Therefore, it could be beneficial for future studies to identify if provider specific characteristics, like these, are related to transition readiness in youth with T1D.

An additional methodological factor that may have impacted the results of the study is the small sample of providers. The provider sample was constricted in size due to the single site for recruitment, with an average diabetes care team consisting of only 5 providers (i.e., endocrinologist, dietitian, psychologist, diabetes educator, diabetes nurse). As a result, all participants included in the analysis receive their medical treatment from the same select providers. The high reports of satisfaction reported by both parents and adolescents may be provider specific and therefore, the results may not generalize to other medical centers or healthcare providers. Another methodological factor that may have impacted the results of the study include the high refusal rate (44\%) and subsequent selectivity bias of the participants. Though all eligible adolescents were approached to participate in the study, the participants who chose to participate in the study may have differing characteristics than those who refused participation. For example, individuals who chose to participate may have had a more positive experience with the medical system or with their provider. Therefore, the results of the study may not be representative of all adolescents' clinic experiences.

Our results further showed that glycemic control does not appear to mediate the relationship between provider-patient communication and transition readiness among adolescents with T1D. Again, these results may suggest that there are other possible factors, outside of provider-patient communication, that significantly impact glycemic control. For example, research identifies that puberty status impacts glycemic control, with the onset of puberty leading to insulin resistance among adolescents (Amiel et al., 1986; Cho, Craig \& Donaghue, 2014). 
Research has found puberty to reduce individual's glucose metabolism, resulting in insulin resistance (Amiel et al., 1986). Though puberty induced insulin resistance has been observed for individuals with and without T1D, the impact of insulin resistance on adolescents with insulindependent diabetes makes it difficult for adolescents with T1D to maintain optimal glycemic control (Amiel et al., 1986). Indeed, our study involved adolescents aged 13 to 17 years; thus, puberty status likely varied across our sample, though this was not measured. Given our study design, we were not able to determine the role that pubertal status might have played in our analyses and results.

Finally, another factor that could predict glycemic control is clinician and family support for adolescent autonomy. Williams and colleagues (2005) found that, for adults, patient autonomy was associated with improved glycemic control. Yet, research with adolescents with T1D suggests that parental involvement helps promote adherence and disease management (e.g., Palmer et al., 2010). Hence, patient autonomy may be an important factor to measure when predicting adolescent transition readiness. Thus, future research of adolescent transition readiness should assess patient autonomy, to determine if it is a better predictor of transition readiness. Validated measures, such as the Perceived Competence for Diabetes Scale (PCDS), could be used to measure T1D specific autonomy (Williams, Freedman, \& Deci, 1998).

Moreover, results from the current study suggests that transition readiness is multifaceted, and it may be necessary to predict it using a complex model of several predictors including patient autonomy.

\section{Strengths}

The current study had several strengths, both methodologically and empirically, which deserve mention. One strength is the collection of both parent-report and child-report of 
perspectives on provider-patient communication. Frequently, pediatric and child research relies on parent report when assessing psychosocial constructs of pediatric patients; yet, perceptions of the end user (i.e., adolescent) of that communication are lacking if researchers do not consider their perspectives as well. Therefore, a strength of our study was the inclusion of both parents and adolescents in providing provider-patient communication, which allowed for the study to better understand both parent and adolescent perspective of satisfaction with provider communication. A second strength of the current study is the method of data collection, where responses were collected in clinic, immediately after the patient interacted with their provider. This timing may have improved reliability and validity of communication ratings, with respondents less likely to be impacted by memory biases or gaps.

The current study also used psychometrically tested measures, which have been determined to have acceptable validity and reliability when used with adolescent populations. The use of such measures made it possible to more accurately measure psychological constructs (i.e., satisfaction with provider-patient communication, transition readiness) and strengthens the overall validity of the study. Finally, the current study measured provider-patient communication and transition readiness across the age range of adolescence (i.e., 13 to 17 years old). Since adolescence has been identified as an important period to study in relation to disease management, the study was able to highlight differences across this developmental period.

\section{Limitations}

The current study had several limitations that potentially limit its generalizability and interpretation. First, all measures, outside of glycemic control, were self-report, which may not be valid characterizations of provider-patient communication or transition readiness. The reliance on self-report measures may explain partially why non-significant results were observed. 
Additionally, provider-patient communication is a complex, bidirectional interaction. The interaction of the provider-patient dyad influences individual behaviors of both the patient and the provider. Therefore, the adolescent's behavior and degree of communication likely impacts the quality and content of the provider's communication and vice versa. Research by Sonneveld and colleagues (2013) found that pediatric providers did not frequently involve the adolescent in medical decision making or see the adolescent without a parent present. When looking at the bidirectional relationship of provider-patient communication, when providers do not actively engage the adolescent patient, the patient in turn may be less involved in their clinic visits and diabetes care. Indeed, informal clinic observations suggest that many adolescent patients do not actively participate in their medical appointment (e.g., focus on cell phone during visit, not ask physician questions regarding treatment), which in turn may lead to less direct physician interaction and reduced provider-patient communication. As a result, the complexities of the provider-patient interaction may require a more robust measurement tool in order to accurately quantify it.

A second limitation is the homogeneous sample. As noted previously, participants in the study were 96\% Caucasian, from an Appalachian population. Further, all participants were recruited from one medical hospital and received services from a small set of medical providers (i.e., five total providers). The lack of representation of race and ethnicities across both the participants and the providers may not represent provider-patient communication among other diverse groups. Finally, other sample characteristics, such as patient self-efficacy, were not addressed in the current study. Yet, these factors could impact adolescent's glycemic control and transition readiness above provider-patient communication alone. 
An additional limitation of the current study is the semantics used in the Mind The Gap assessment measure. When assessing adolescents' and parents' perception of provider communication, this measure refers to all medical staff broadly. The measure's general assessment of medical staff's quality of communication does not permit analyzing variation in perceptions of communication across individual medical team members (e.g., physician, dietician, nurse, psychologist). Future studies of provider-patient communication should look to assessing perception of communication quality for specific or individual team members. Additionally, this measure of communication does not use reverse coding for some items, resulting in potential response bias stemming from social desirability among participants. The response format may explain the ceiling effect that was observed on parent and adolescent reports of provider communication. Indeed, another study using the Mind The Gap questionnaire also reported high levels of adolescent and parent satisfaction with provider-patient communication with median item response scores equal to or above a 4 (Sonneveld et al., 2013). The current study observed an even greater level of adolescent- and parent-reported providerpatient communication satisfaction with median item response scores equal to or above a 4.5.

An important final point to note, and a potential limitation of the current study, is that the mediation analysis was run using cross-sectional data. This analysis implies that change in provider-patient communication would impact change in glycemic control, and change in glycemic control would impact change in transition readiness. However, because the data are cross-sectional in nature, rather than longitudinal, this interpretation cannot necessarily be made.

\section{Implications}

Although the current study did not find a significant relationship between providerpatient communication and transition readiness, these findings are important to consider in 
understanding transition readiness and provide some direction for future research. Past research has identified the impact transition readiness has on health behavior for adolescents with T1D (e.g., frequency of clinic visits, filling prescriptions, monitoring blood sugar levels). Given the health implications transition readiness has, it is important to optimize adolescents' transition readiness through behavior health interventions. However, results from the current study indicate that provider-patient communication alone may not be sufficient for improving transition readiness. Moreover, there may be additional variables in addition to glycemic control that account for variability in the relationship between provider-patient communication and transition readiness. Ultimately, factors influencing transition readiness among adolescents with T1D are likely more complex, and could not be fully understood given the simplicity of the study.

The study also revealed that parent-report and adolescent self-report of provider-patient communication were not significantly correlated. This finding implies that adolescents and their parents have different experiences interacting with providers. These results are consistent with other studies that have found adolescents to be less satisfied with their providers listening skills, understanding, and honestly, when compared to parent reports (Sonneveld et al., 2013). It may be important for future studies to look at parent and adolescent reports separately to ensure that all points of view are understood.

\section{Future Research}

Given the impact transition readiness has on health behavior, future research should continue to look at factors that impact transition readiness among adolescents with T1D, in order to identify ways to improve the transition process and subsequent health behaviors. Future studies should continue to evaluate relationships between provider-patient communication and transition readiness using more robust measures. Additionally, factors that impact transition 
readiness should be assessed more broadly, using a more heterogeneous sample of patients and providers. Additional variables, such as duration of T1D, time of T1D onset, and adolescent selfefficacy should also be assessed to determine their impact on transition readiness. Additionally, future research should evaluate predictors of transition readiness that are not self-report measures. Potential methods to use include behavioral observation and qualitative coding of the provider-patient interaction. Such methods would eliminate the potential self-report biases that are common among self-report measures. Finally, future research should investigate other predictors of transition readiness outside of provider-patient communication. Identifying factors that impact transition readiness may aid in the potential development of behavioral health interventions for adolescents with T1D.

\section{Conclusion}

Transition readiness is an important area of study within pediatric psychology, and potential factors to optimize transition should be investigated systematically, and in turn, health outcomes for adolescence with T1D. Results from the current study do not indicate that providerpatient communication influences transition readiness among adolescence with T1D. Further, glycemic control was not found to mediate or moderate this relationship. Therefore, future research should continue to explore potential factors that are associated with transition readiness, including patient self-efficacy, provider characteristics, and duration of diabetes onset. Additionally, future research should utilize behavioral observation and qualitative coding when assessing social constructs. Finally, research should assess the impact race and ethnicity has on the transition process, in order to promote adequate diabetes health outcomes across race and ethnicity and to reduce health disparities in minority populations. Though a relationship between provider-patient communication and transition readiness was not found, the study may help 
guide future research when investigating other factors that impact transition readiness among adolescents with a chronic illness. 


\section{References}

Abdul-Rasoul, M., Habib, H., \& Al-Khouly, M. (2006). "The honeymoon phase" in children with type 1 diabetes mellitus: Frequency, duration, and influential factors. Pediatric Diabetes, 7(2), 101-107.

American Academy of Pediatrics, American Academy of Family Physicians, American College of Physicians, Transitions Clinical Report Authoring Group. (2011). Supporting the health care transition from adolescence to adulthood in the medical home. Pediatrics, $128,182-$ 200.

American Diabetes Association. (2015a). Food \& fitness. Retrieved from http://www.diabetes.org/food-and- fitness/

American Diabetes Association. (2015b). DKA (Ketoacidosis) \& ketones. Retrieved from http://www.diabetes.org/ living-with-diabetes/complications/ketoacidosis-dka.html American Diabetes Association. (2015c). Hypoglycemia (low blood glucose). Retrieved from http://www.diabetes.org/living-with-diabetes/treatment-and-care/blood-glucosecontrol/hypoglycemia-low-blood

American Diabetes Association. (2017). Standards of medical care in diabetes-2017. Diabetes Care, 40, S1-S135.

Amiel, S. A., Sherwin, R. S., Simonson, D. C., Lauritano, A. A., \& Tamborlane, W. V. (1986). Impaired insulin action in puberty. New England Journal of Medicine, 315(4), 215-219. doi:10.1056/NEJM198607243150402

Armstrong, K., Ravenell, K., McMurphy, S., Putt, M. (2007). Racial/ethnic differences in physician distrust in the United States. American Journal of Public Health, 97, 1283 1289. 
Aronson, J., Burgess, D., Phelan, S. M., \& Juarez, L. (2013). Unhealthy interactions: The role of stereotype threat in health disparities. American Journal of Public Health, 103(1), 50-56.

Blum, R., Garell, D., Hodgman, C. H., Jorissen, T. W., Okinow, N. A. Orr, D. P. \& Slap, G. B. (1993). Transition from child-centered to adult health-care systems for adolescents with chronic conditions: A position paper of the society for adolescent medicine. Journal of Adolescent Health, 14, 570-576.

Bryden, K., Peveler, R., Stein, A., Neil, A., Mayou, R., \& Dunger, D. (2001). Clinical and psychological course of diabetes from adolescence to young adulthood: A longitudinal cohort study. Diabetes Care, 24, 1536-1540.

Cadario, F., Prodam, F., Bellone, S., Trada, M., Binotti, M., Allochis, G., Baldelli, R.,.... \& Aimaretti, G. (2009). Transition process of patients with type diabetes (T1DM) from paediatric to the adult health care service: A hospital-based approach. Clinical Endocrinology, 71, 346-350.

Campbell, C., Lockyer, J., Laidlaw, T., \& Macleod, H. (2007). Assessment of a matched-pair instrument to examine doctor-patient communication skills in practising doctors. Medical Education, 41(2), 123-9.

Cervia, J. S. (2013). Easing the transition of HIV-infected adolescents to adult care. AIDS Patient Care and STDs, 27(12), 692-696.

Cho, Y. H., Craig, M. E., \& Donaghue, K. C. (2014). Puberty as an accelerator for diabetes complications. Pediatric Diabetes, 15(1), 18-26. 
Dabelea, D., Mayer-Davis, E. J., Saydah, S., Imperatore, G., Linder, B., Divers, J.,.... \& Hamman, R. F. (2014). Prevalence of type 1 and type 2 diabetes among children and adolescents from 2001 to 2009. The Journal of the American Medical Association, 311, 1778-1786.

The Endocrine Society. (2013). Managing the transition of care for patients with type 1 diabetes. Retrieved from https://www.endocrine.org/education-and-practice- management/practicemanagement-resources/clinical-practice-resources/ transition-of-care.

Erdfeld, E., Faul, F., \& Buchner, A. (1996). GPOWER: A general power analysis program. Behavior Research Methods, Instruments, \& Computers, 28, 1-11.

Ferris, M., Harward, D., Bickford, K., Layton, J., Ferris, M., Hogan, S., Gipson, D., McCoy, L., Hooper, S. (2012). A clinical tool to measure the components of healthcare transition from pediatric care to adult care: the UNC TR(x)ANSITION scale. Renal Failure, 34, $744-753$.

Fleming, E., Carter, B., \& Gillibrand, W. (2002). The transition of adolescents with diabetes from the children's health care service into the adult health care service: A review of the literature. Journal of Clinical Nursing, 11, 560-567.

Garvey, K. C., Telo, G. H., Needleman, J. S., Forbes, P., Finkelstein, J. A., \& Laffel, L. M. (2016). Health care transition in young adults with type 1 diabetes: Perspectives of adult endocrinologists in the U.S. Diabetes Care, 39(2), 190-197.

Garvey, K. C., Wolpert, H. A., Rhodes, E. T., Laffel, L. M., Kleinman, K., Beste, M. G., Wolfsdorf, J. I., \& Finkelstein, J. A. (2012). Health care transition in patients with type 1 diabetes. Diabetes Care, 35(8), 1716-1722. 
Hayes, A. F. (2017). Introduction to mediation, moderation, and conditional process analysis: A regressio- based approach. Guilford Publications.

Javalkar, K., Johnson, M., Kshirsagar, A. V., Ocegueda, S., Detwiler, R. K., \& Ferris, M. (2016). Ecological factors predict transition readiness/self-management in youth with chronic conditions. Journal of Adolescent Health, 58(1), 40-46.

Jeffcoate, S. L. (2004). Diabetes control and complications: The role of glycated haemoglobin, 25 years on. Diabetic Medicine, 21, 657-665.

Jensen, P. T., Paul, G. V., LaCount, S., Peng, J., Spencer, C. H., Higgins, G. C., ... Ardoin, S. P. (2017). Assessment of transition readiness in adolescents and young adults with chronic health conditions. Pediatric Rheumatology, 15(1), 1-7.

Krupat, E., Frankel, R., Stein, T., Irish, J. (2006). The four habits coding scheme: Validation of an instrument to assess clinicians' communication behavior. Patient Education Counsil., $62(1), 38-45$.

Lancaster, B. M., Gadaire, D. M., Holman, K., \& Leblanc, L. A. (2015). Association between diabetes treatment adherence and parent-child agreement regarding treatment responsibilities. Family Systems Health, 33(2), 120-125.

Law, G. U., Kelly, T. P., Huey, D., \& Summerbell, C. (2002). Self-management and well-being in adolescents with diabetes mellitus: Do illness representations play a regulatory role? Journal of Adolescent Health, 31, 381-385.

Logan, J., Peralta, E., Brown, K., Moffett, M., Advani, A., \& Leech, N. (2008). Smoothing the transition from paediatric to adult services in type 1 diabetes. Diabetes Nursing, 12, 328338. 
Lotstein, D. S., Seid, M., Klingensmith, G., Case, D., Lawrence, J. M., ... \& Pihoker, C. (2013). Transition from pediatric to adult care for youth diagnosed with type 1 diabetes in adolescence. Pediatrics, 131(4), e1062 LP-e1070.

Lugasi, T., Achille, M., \& Stevenson, M. (2011). Patients' perspective on factors that facilitate transition from child-centered to adult-centered health care: A theory integrated metasummary of quantitative and qualitative studies. Journal of Adolescent Health, 48(5), 429-440.

Lyons, S. K., Libman,I. M., \& Sperling, M. A. (2013). Diabetes in the adolescent: Transitional issues. The Journal of Clinical Endocrinology \& Metabolism, 98(12), 4639-4645.

Mayer-Davis, E. J., Lawrence, J. M., Dabelea, D., Divers, J., Isom, S., Dolan, L., ... Wagenknecht, L. (2017). Incidence trends of type 1 and type 2 diabetes among youths, 2002-2012. New England Journal of Medicine, 376(15), 1419-1429.

Mellin, A. E., Neumark-Sztainer, D., Patterson, J., \& Sockalosky, J. (2004). Unhealthy weight management behavior among adolescent girls with type 1 diabetes mellitus: The role of familial eating patterns and weight-related concerns. Journal of Adolescent Health, 35(4), 278-89.

Moens, A., Grypdonck, M. H. F., \& Van, D. B. (2001). The development and psychometric testing of an instrument to measure diabetes management self-efficacy in adolescents with type 1 diabetes. Scholarly Inquiry for Nursing Practice, 15(3), 223-233.

Monaghan, M., Hilliard, M., Sweenie, R., \& Riekert, K. (2013). Transition readiness in adolescents and emerging adults with diabetes: The role of provider-patient communication. Current Diabetes Reports, 13(6), 900-908. 
Palmer, D. L., Berg, C. A., Butler, J., Fortenberry, K., Murray, M., Lindsay, R., ... \& Wiebe, D. J. (2008). Mothers', fathers', and children's perceptions of parental diabetes responsibility in adolescence: Examining the roles of age, pubertal status, and efficacy. Journal of Pediatric Psychology, 34(2), 195-204.

Palmer, D. L., Osborn, P., King, P. S., Berg, C. A., Butler, J., Butner, J., Horton, D., ... Wiebe, D. J. (2010). The structure of parental involvement and relations to disease management for youth with type 1 diabetes. Journal of Pediatric Psychology, 36(5), 596-605.

Patterson, D. L., \& Lanier, C. (1999). Adolescent health transitions: Focus group study of teens and young adults with special health care needs. Family \& Community Health: The Journal of Health Promotion \& Maintenance, 22(2), 43-58.

Peek, M. E., Quinn, M. T., Gorawara-Bhat, R., Odoms-Youn, A., Wilson, S. C., \& Chin, M. H. (2008). How is shared decision-making defined among African Americans with diabetes? Patient Education and Counseling, 72, 450-8.

Pendley, J. S., Kasmen, L. J., Miller, D. L., Donze, J., Swenson, C., \& Reeves, G. (2002). Peer and family support in children and adolescents with type 1 diabetes. Journal of Pediatric Psychology, 27(5), 429-38.

Peters, A., \& Laffel, L. (2011). Diabetes care for emerging adults: Recommendations for transition from pediatric to adult diabetes care systems. Diabetes Care, 34(11), 24772485.

Petitti, D. B., Klingensmith, G. J., Bell, R. A., Andrews, J. S., Dabelea, D., Imperatore, G., ... Mayer-Davis, E. (2009). Glycemic control in youth with diabetes: The SEARCH for diabetes in youth study. Journal of Pediatrics, 155(5), 668-672. 
Preacher, K. J., \& Hayes, A. F. (2008). Asymptotic and resampling strategies for assessing and comparing indirect effects in multiple mediator models. Behavior Research Methods, 40(3), 879-891.

Price, C. S., Corbett, S., Lewis-Barned, N., Morgan, J., Oliver, L. E., \& Dovey-Pearce, G. (2011). Implementing a transition pathway in diabetes: A qualitative study of the experiences and suggestions of young people with diabetes. Child: Care, Health and Development, 37(6), 852-860.

Rao, J. K., Anderson, L. A., Sukumar, B., Beauchesne, D. A., Stein, T., \& Frankel, R. M. (2010). Engaging communication experts in a Delphi process to identify patient behaviors that could enhance communication in medical encounters. BMC Health Services Research, 10(1), 97-110.

Ritholz, M. D., Wolpert, H., Beste, M., Atakov-Castillo, A., Luff, D., \& Garvey, K. C. (2014). Provider-patient relationships across the transition from pediatric to adult diabetes care: A qualitative study. The Diabetes Educator, 40(1), 40-47.

Rosen, D., Blum, R., Britto, M., Sawyer, S., \& Siegel, D. (2003). Transition to adult health care for adolescents and young adults with chronic illness. Journal of Adolescent Health, 33, $309-311$.

Sawicki, G. S., Lukens-Bull, K., Yin, X., Demars, N., Huang, I., Livingood, W., . . \& Wood, D. (2011). Measuring the transition readiness of youth with special healthcare needs: Validation of the TRAQ_-Transition Readiness Assessment Questionnaire. Journal of Pediatric Psychology, 36, 160-171.

Schidlow, D., \& Fiel, S. (1990). Transition of chronically ill adolescents from pediatric to adult health care systems. Medical Clinics of North America, 74, 1113-1120. 
Shaw, K. L., Southwood, T. R., \& McDonagh, J. E. (2007). Development and preliminary validation of the Mind the Gap scale to assess satisfaction with transitional health care among adolescents with juvenile idiopathic arthritis. Child: Care, Health and Development, 33(4), 380-388.

Silverstein, J., Klingensmith, G., Copeland, K., Plotnick, L., Kaufman, F., Laffel, L., ... \& Clark, N. (2005). Care of children and adolescents with type 1 diabetes. Diabetes Care, 28(1), $186-212$.

Simms, M., Baumann, K., \& Monaghan, M. (2017). Health communication experiences of emerging adults with type 1 diabetes. Clinical Practice in Pediatric Psychology, 5(4), 415-425.

Sonneveld, H. M., Strating, M. M. H., Van Staa, A. L., \& Nieboer, A. P. (2013). Gaps in transitional care: What are the perceptions of adolescents, parents and providers? Child: Care, Health and Development, 39(1), 69-80.

Stinson, J., Kohut, S .A., Spiegel, L., White, M., Gill, N., Colbourne, G., Sigurdson, S., Duffy, K. W., Tucker, L., Stringer, E., Hazel, B., Hochman, J., Reiss, J., \& Kaufman, M. (2014). A systematic review of transition readiness and transfer satisfaction measures for adolescents with chronic illness. International Journal of Adolescent Medicine and Health, 26, 1-16.

Street, R. L. J., Makoul, G., Arora, N. K., \& Epstein, R. M. (2009). How does communication heal? Pathways linking clinician-patient communication to health outcomes. Patient Education and Counseling, 74, 295-301.

Teal, C., Street, R. (2009). Critical elements of culturally competent communication in the medical encounter: A review and model. Social Science and Medicine, 68, 533-43. 
Thoemmes, F., MacKinnon, D. P., \& Reiser, M. R. (2010). Power analysis for complex mediational designs. Structural Equation Modeling, 17(3), 510-534.

Van Staa, A. L., Jedeloo, S., van Meeteren, J., \& Latour, J. M. (2011). Crossing the transition chasm: Experiences and recommendations for improving transitional care of young adults, parents and providers. Child: Care, Health and Development, 37(6), 821-832.

Van Staa, A. L., Van der Stegege, H. A., Jedeloo, S., Moll, H. A., \& Hilberink, S. (2010). Readiness to transfer to adult care of adolescents with chronic conditions: Exploration of associated factors. Journal of Adolescent Health, 48(2011), 295-302.

Vanelli, M., Caronna, S., Adinolfi, B., Chiari, G., Gugliotta, M., \& Arsenio, L. (2004). Effectiveness of an uninterrupted procedure to transfer adolescents with type 1 diabetes from the paediatric to the adult clinic held in the same hospital: Eight-year experience with the Parma protocol. Diabetes, Nutrition, and Metabolism, 17, 304-308.

Visentin, K., Koch, T., \& Kralik, D. (2006). Adolescents with type 1 diabetes: Transition between diabetes services. Journal of Clinical Nursing, 15(6), 761-769.

Walleghem, N. V., MacDonald, C. A., \& Dean, H. J. (2008). Evaluation of a systems navigator model for transition from pediatric to adult care for young adults with type 1diabetes. Diabetes Care, 31(8), 1529-1530.

Weissberg-Benchell, J., Wolpert, H., \& Anderson, B. J. (2007). Transitioning from pediatric to adult care. Diabetes Care, 30(10), 2441-2446.

Williams, G. C., Freedman, Z. R., \& Deci, E. L. (1998). Supporting autonomy to motivate patients with diabetes for glucose control. Diabetes Care, 21, 44-51. 
Williams, T., Sherman, E., Dunseith, C., Mah, J., Blackman, M., Latter, J., Mohamed, I., Slick, D., Thornton, N. (2010). Measurement of medical self-management and transition readiness among Canadian adolescents with special health care needs. International Journal of Child and Adolescent Health, 3, 527-535.

Wills, C., Scott, A., Swift, P., Davies, M., Mackie, A., \& Mansell, P. (2003). Retrospective review of care and outcomes in young adults with type 1 diabetes. British Medical Journal, 327, 260-261.

Wood, D. L., Sawicki, G. S., Miller, D., Smotherman, C., Lukens-Bull, K., Livingood, W. C., . . \& Kraemer, D. F. (2014). The transition readiness assessment questionnaire (TRAQ): Its factor structure, reliability, and validity. Academic Pediatrics, 14, 415-422.

Wood, J. R., Miller, K. M., Maahs, D. M., Beck, R. W., DiMeglio, L. A., Libman, I. M., Quinn, M.,...\& Woerner, S. E. (2013). Most youth with type 1 diabetes in the T1D exchange clinic registry do not meet American Diabetes Association or International Society for pediatric and adolescent diabetes clinical guidelines. Diabetes Care, 36, 2035- 2037.

Zhang, L. F., Ho, J. S., \& Kennedy, S. E. (2014). A systematic review of the psychometric properties of transition readiness assessment tools in adolescents with chronic disease. BioMed Central Pediatrics, 14(4), 1-10

Zhou, H., Roberts, P., Dhaliwal, S., \& Della, P. (2016). Transitioning adolescent and young adults with chronic disease and/or disabilities from paediatric to adult care services - an integrative review. Journal of Clinical Nursing, 25(21-22), 3113-3130. 


\section{Tables and Figures}

Table 1

Child and Parent Demographics

\begin{tabular}{|c|c|c|}
\hline \multicolumn{3}{|c|}{ M (SD)/n (\%) } \\
\hline Characteristic & $\begin{array}{c}\text { Child } \\
(n=56)\end{array}$ & $\begin{array}{l}\text { Parent } \\
(n=56)\end{array}$ \\
\hline Age & $15.49(4.0)$ & $43.71(8.4)$ \\
\hline \multicolumn{3}{|l|}{ Race } \\
\hline White/Caucasian & $54(96.5 \%)$ & \\
\hline American Indian/Alaska Native & $1(1.8 \%)$ & \\
\hline Asian & $0(0.0 \%)$ & \\
\hline Black/African-American & $1(1.8 \%)$ & \\
\hline Native Hawaiian/Other Pacific & $0(0.0 \%)$ & \\
\hline \multicolumn{3}{|l|}{ Islander } \\
\hline Other & $1(1.8 \%)$ & \\
\hline Hispanic/Latino & $0(0.0 \%)$ & \\
\hline Female & $25(43.9 \%)$ & $48(84.2 \%)$ \\
\hline Caregiver (Mother) & & $46(80.7 \%)$ \\
\hline \multicolumn{3}{|l|}{ Highest Education Level } \\
\hline Some High School & & $0(0.0 \%)$ \\
\hline High School/GED & & $15(26.3 \%)$ \\
\hline Some College & & $21(36.8 \%)$ \\
\hline Bachelor's & & $12(21.1 \%)$ \\
\hline Master's/Doctoral & & $9(15.8 \%)$ \\
\hline \multicolumn{3}{|l|}{ Marital Status } \\
\hline Single & & $3(5.3 \%)$ \\
\hline Divorced & & $9(15.8 \%)$ \\
\hline Married to other biological parent & & $35(61.4 \%)$ \\
\hline Remarried to step-parent & & $6(10.5 \%)$ \\
\hline Living with boyfriend/girlfriend & & $1(1.8 \%)$ \\
\hline Widowed & & $1(1.8 \%)$ \\
\hline Other & & $0(0.0 \%)$ \\
\hline \multicolumn{3}{|l|}{ Income } \\
\hline$<\$ 10,000$ & & $2(3.5 \%)$ \\
\hline$\$ 10,000-\$ 14,999$ & & $2(3.5 \%)$ \\
\hline$\$ 15,000-\$ 24,999$ & & $2(3.5 \%)$ \\
\hline$\$ 25,000-\$ 34,999$ & & $5(8.8 \%)$ \\
\hline$\$ 35,000-\$ 49,999$ & & $5(8.8 \%)$ \\
\hline$\$ 50,000-\$ 74,999$ & & $5(8.8 \%)$ \\
\hline$\$ 75,000-\$ 99,999$ & & $10(17.5 \%)$ \\
\hline$\$ 100,000-\$ 149,999$ & & $11(19.3 \%)$ \\
\hline$>\$ 150,000$ & & $7(12.3 \%)$ \\
\hline \# of appointments in past 12 months & $1.97(.9)$ & \\
\hline HbAlc & $8.65(1.8)$ & \\
\hline TID Duration (years) & $6.37(3.9)$ & \\
\hline
\end{tabular}


T1D Regimen

Multiple daily insulin Injection

$20(35.1 \%)$

Insulin pump

$31(54.4 \%)$

Twice daily insulin

$0(0.0 \%)$

Basal/bolus insulin

$1(1.8 \%)$

Multiple treatment

$5(8.8 \%)$

Insurance Status

Private

$41(71.9 \%)$

Public/Medicaid

$16(28.1 \%)$ 
Table 2

Descriptive Statistics and Correlations among Covariates and Variables

\begin{tabular}{|c|c|c|c|c|c|c|c|c|c|c|c|c|}
\hline 11 & $n$ & $M(S D)$ & Range & 1 & 2 & 3 & 4 & 5 & 6 & 7 & 8 & 9 \\
\hline 1. Age & 56 & $15.5(1.50)$ & $13-17$ & --- & & & & & & & & \\
\hline 2. Duration & 56 & $6.3(3.88)$ & $1-16$ & $.29 *$ & --- & & & & & & & \\
\hline 3. Parent MTG- PC & 56 & $6.6(.46)$ & $5.4-7.0$ & .02 & -.14 & $\begin{array}{l}-- \\
\end{array}$ & & & & & & \\
\hline 4. Adol MTG - PC & 56 & $6.3(.74)$ & $4.0-7.0$ & -.10 & .24 & .06 & --- & & & & & \\
\hline 5. Parent MTG & 56 & $6.3(.62)$ & $4.6-7.0$ & -.06 & -.04 & $.89 * *$ & .18 & --- & & & & \\
\hline 6. Adol MTG & 56 & $6.2(.84)$ & $3.7-7.0$ & -.16 & .19 & .06 & $.94 * *$ & .18 & --- & & & \\
\hline 7. TRAQ (No Com) & 56 & $3.2(.92)$ & $1.7-5.0$ & $.42 * *$ & .10 & .08 & -.12 & .10 & -.11 & --- & & \\
\hline 8. TRAQ & 56 & $3.4(.83)$ & $2.0-5.0$ & $.41 * *$ & .10 & .08 & -.11 & .09 & -.10 & $.99 * *$ & --- & \\
\hline 9. $\mathrm{HbAlc}$ & 56 & $8.6(1.8)$ & $5.7-13.8$ & -.16 & $.35^{*}$ & -.43 & .11 & -.27 & .10 & -.04 & -.04 & --- \\
\hline
\end{tabular}

Note. Parent MTG-PC = Parent Mind the Gap-Provider Characteristics subscale; Adol MTG-PC = Adolescent Mind the GapProvider Characteristics subscale; Parent MTG = Parent Mind the Gap Questionnaire; Adol MTG = Adolescent Mind the Gap Questionnaire; TRAQ (No Com) = Transition Readiness Assessment Questionnaire without Communication subscale; TRAQ = Transition Readiness Assessment Questionnaire; HbA1c = glycemic control; Duration T1D= Duration of time in years diagnosed with T1D; Higher values on MTG and TRAQ scales indicate more positive responses; Higher values on HbA1c indicate less diabetes control.. $*=p<.05, * *=p<.001$ 
Table 3

Unstandardized Coefficients and Standard Errors for Regression Models Predicting Transition Readiness

\begin{tabular}{lccc}
\hline Variable & \multicolumn{2}{l}{ Unstandardized Beta (SE) } & \multicolumn{2}{c}{ Model Indices } \\
\hline Model 1: Adolescent reported provider-patient communication and glycemic control \\
\hline Age & $-0.002(.03)$ & $F$-statistic & 0.63 \\
Duration T1D & $0.019(.04)$ & $d f$ & $(5,41)$ \\
Adolescent-MTG & $0.94(.66)$ & $R^{2}$ & 0.072 \\
HbA1c & $0.72(.47)$ & & \\
Adolescent MTG x & $-0.12(.07)$ & & \\
HbA1c & & & \\
\hline
\end{tabular}

Model 2: Parent reported provider-patient communication and glycemic control

\begin{tabular}{lccc}
\hline Age & $0.003(.03)$ & $F$-statistic & 0.21 \\
Duration T1D & $0.008(.04)$ & $d f$ & $(4,41)$ \\
Parent-MTG & $0.72(1.13)$ & $R^{2}$ & 0.025 \\
HbA1c & $0.36(.76)$ & & \\
Parent MTG x HbA1c & $-0.059(.12)$ & & \\
\hline
\end{tabular}

Model 3: Adolescent reported provider-patient communication with Provider Characteristics subscale and glycemic control

\begin{tabular}{lccc}
\hline Age & $-0.002(.03)$ & & \\
Duration T1D & $0.019(.04)$ & $F$-statistic & 0.57 \\
Adolescent-MTG-PC & $0.96(.82)$ & $d f$ & $(5,41)$ \\
HbA1c & $0.81(.60)$ & $R^{2}$ & 0.065 \\
Adolescent MTG-PC x & $-0.13(.09)$ & & \\
HbAlc & $-0.002(.03)$ & $F-$ statistic & 0.14 \\
Model 4: Parent reported provider-patient communication with Provider Characteristics \\
subscale and glycemic control & $0.009(.04)$ & $d f$ & $(5,41)$ \\
Age & $1.19(1.75)$ & $R^{2}$ & 0.017 \\
Duration T1D & $0.67(1.13)$ & & \\
Parent-MTG-PC & $-0.10(.17)$ &
\end{tabular}


Note. Regressions computed using Hayes PROCESS. Parent MTG-PC = Parent Mind the GapProvider Characteristics subscale; Adol MTG-PC = Adolescent Mind the Gap-Provider Characteristics subscale; Parent MTG = Parent Mind the Gap Questionnaire; Adol MTG = Adolescent Mind the Gap Questionnaire; HbA1c = glycemic control; Duration T1D= Duration of time in years diagnosed with T1D. ${ }^{*} p<.05 .{ }^{*} p<.01$. 
Figure 1.

Mediation analysis of adolescent reported provider-patient communication, glycemic control (HbAlc), and levels of transition readiness.

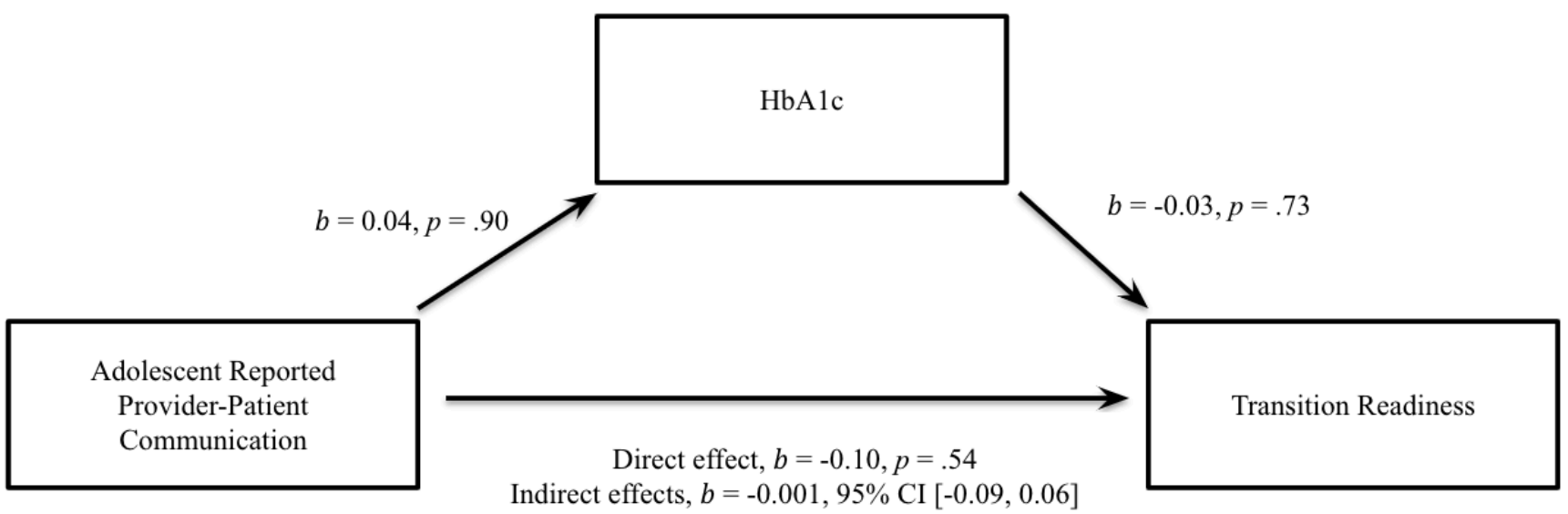


Figure 2 .

Mediation analysis of parent reported provider-patient communication, glycemic control (HbAlc), and levels of transition readiness.

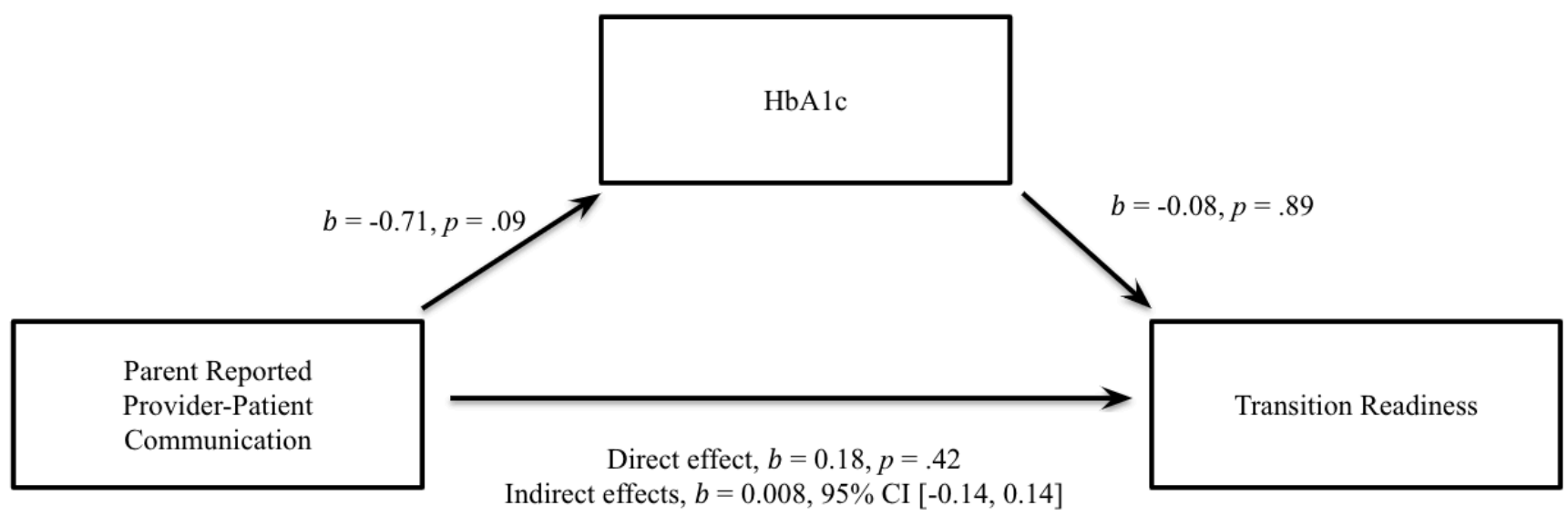


Figure 3 .

Mediation analysis of adolescent reported provider-patient communication with Provider Characteristics subscale, glycemic control (HbAlc), and levels of transition readiness without communication subscale.

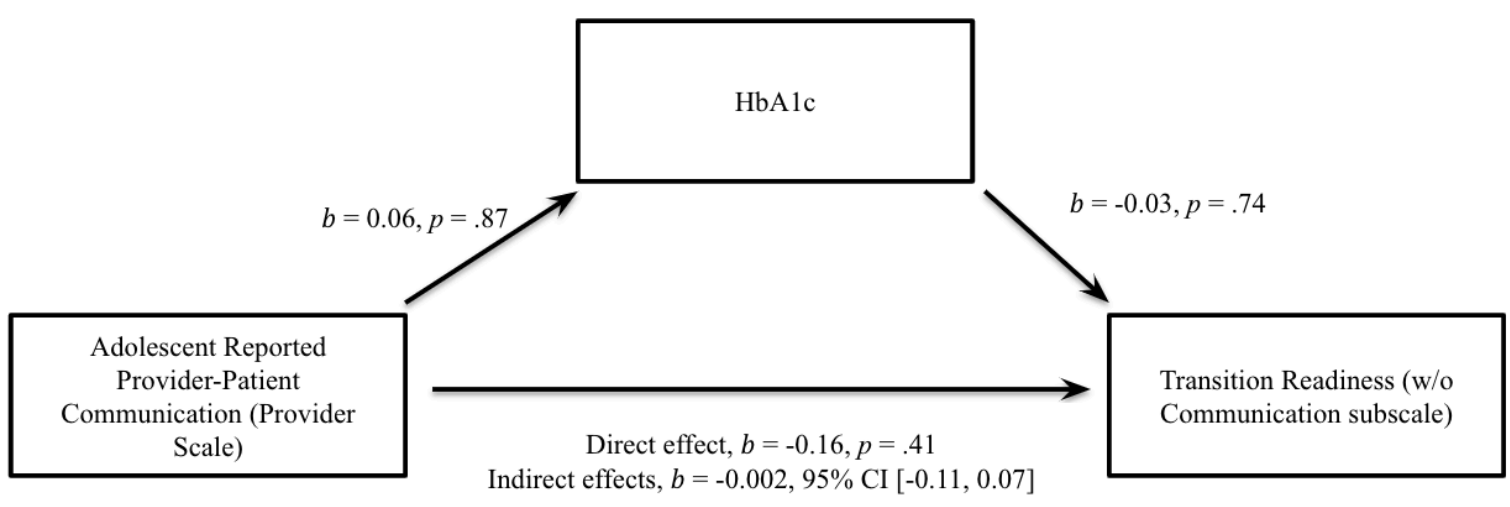


Figure 4.

Mediation analysis of parent reported provider-patient communication with Provider Characteristics subscale, glycemic control (HbAlc), and levels of transition readiness without communication subscale.

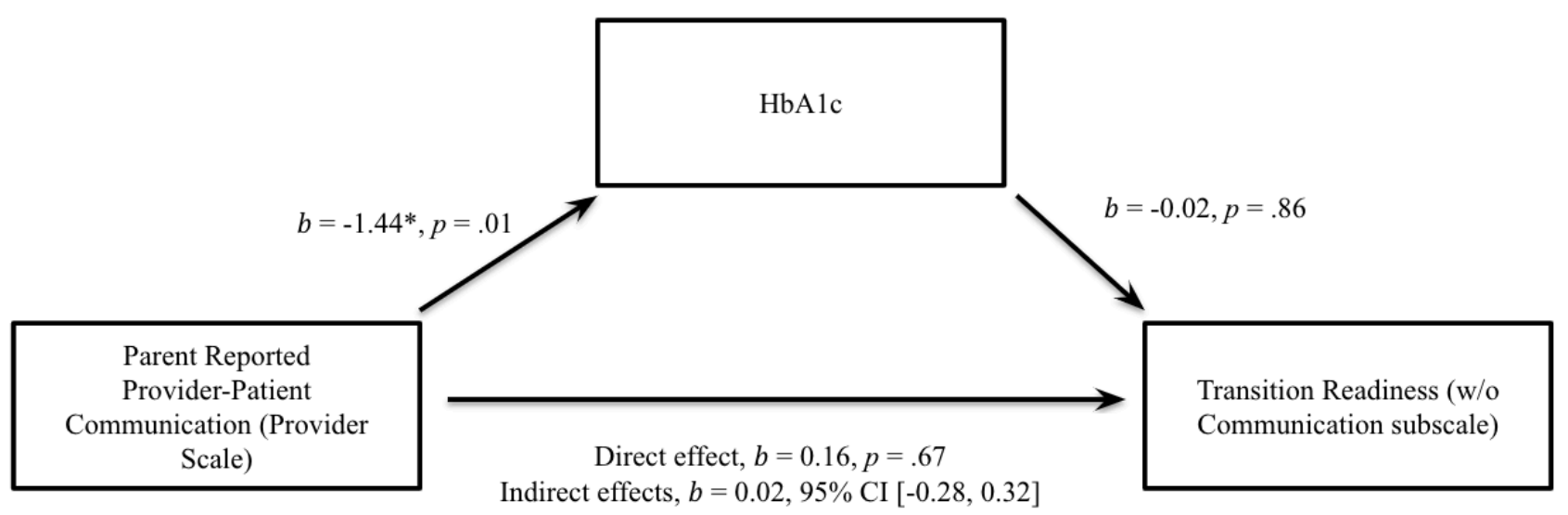

